\title{
Los armónicos que aportan los sistemas fotovoltaicos interconectados a la red un problema latente
}

\section{Harmonics that provide the solar photovoltaic power supply systems interconnected to the electrical grid, are a latent problem}

\author{
DELGADO-PONCE, Luis $\dagger^{*}$, VARGAS-HERNÁNDEZ, Oscar Joel, MARTÍNEZ-MAGDALENO, \\ Arturo y MENÉNDEZ-BENAVENTE, Luis Martín
}

Universidad Tecnológica de Salamanca

ID $1^{\text {er }}$ Autor: Luis, Delgado-Ponce / ORC ID: 0000-0003-0761-1464, Researcher ID Thomson: E-8843-2019, CVU CONACYT ID: 997254

ID $1^{\text {er }}$ Coautor: Oscar Joel, Vargas-Hernández / ORC ID: 0000-0001-6258-4388, CVU CONACYT ID: 335453

ID $2^{\text {do }}$ Coautor: Arturo, Martínez-Magdaleno / ORC ID: 0000-0003-1004-9716, CVU CONACYT ID: 997300

ID $3^{\text {er }}$ Coautor: Luis Martín, Menéndez-Benavente / ORC ID: 0000-0002-4843-3409, CVU CONACYT ID: 997264

DOI: $10.35429 /$ JEE.2019.8.3.5.20

Recibido Febrero 20, 2019; Aceptado Marzo 30, 2019

\section{Resumen}

La energía eléctrica que las generadoras suministran a los usuarios (CFE para México), requiere de especificaciones determinadas para la operación segura y eficiente de equipos y maquinaria. Parte importante de un sistema fotovoltaico interconectado es el inversor y por ser una carga no lineal, inevitablemente distorsionará la onda sinusoidal de voltaje, cuyas componentes pueden ser dañinas para los equipos mencionados, dependiendo del grado de distorsión armónica total que resulte de la operación de estos inversores. El problema de los armónicos originados por los SFVI se agrava por dos situaciones: 1. La tendencia actual del uso de este tipo de sistemas va en aumento, debido a los beneficios, económicos, ambientales y sociales. 2. La forma de conexión conocida como generación distribuida, hace que esté más cerca de los consumidores, es decir los componentes armónicos de la onda distorsionada tendrán mayor impacto. Este estudio de distorsión armónica generada en los inversores es urgente que se analice y se determinen procedimientos y especificaciones para seleccionar el equipo mejor y para minimizar este fenómeno latente, que desafortunadamente no se puede eliminar, pero si reducir sus efectos.

Fotovoltaicos, Inversores, Armónicas

\begin{abstract}
The electric power that generators supply to users (in Mexico, CFE), requires specifications for the safe and efficient operation of equipment and machinery. An important part of an interconnected photovoltaic system is the inverter and because it is a non-linear load, it will inevitably distort the voltage sine wave, whose components can be harmful for the aforementioned equipment, depending on the degree of total harmonic distortion that results from the operation of these investers The problem of the harmonics originated by the SFVI increases because of two situations: 1 . The current trend of the use of this type of systems is increasing, due to the economic, environmental and social benefits. 2. the way of connection for SFVI called distributed generation, produce electricity close to the end users of power, means the harmonic components of the distorted wave will have greater impact. This study of harmonic distortion generated in the invester, it is urgent to analyze and determine procedures and specifications to select the best equipment and to minimize this latent phenomenon, which unfortunately can not be eliminated, but only to reduce its effects.
\end{abstract}

Photovoltaics, Inverters, Harmonics

Citación: DELGADO-PONCE, Luis, VARGAS-HERNÁNDEZ, Oscar Joel, MARTÍNEZ-MAGDALENO, Arturo y MENÉNDEZ-BENAVENTE, Luis Martín. Los armónicos que aportan los sistemas fotovoltaicos interconectados a la red un problema latente. Revista de Ingeniería Eléctrica. 2019. 3-8: 5-20

\footnotetext{
$\dagger$ Investigador contribuyendo como primer Autor.

*Correspondencia al Autor correo electrónico: (luisdelponce@gmail.com)
} 


\section{Introducción}

La calidad de la energía eléctrica se define en términos de las características, instantáneas y a lo largo del tiempo, de sus principales parámetros y variables (basicamente amplitud de la tensión y duración de las variaciones que presenta) y de que estas sean compatibles o no con las que requieren las cargas conectadas a ella para poder funcionar correctamente.

Este concepto de calidad de la energía eléctrica, que se denomina calidad de servicio de la distribución de energía eléctrica, se define como el conjunto de características, técnicas y comerciales, inherentes al suministro eléctrico y exigibles por los consumidores, a las empresas distribuidoras de energía eléctrica.

Algunas de esas características son:

La continuidad de suministro, relativa al número y duración de las interrupciones del suministro eléctrico.

- La calidad del producto, relativa a las características de la onda de tensión.

El análisis de la calidad de la red eléctrica se realiza en el punto de suministro o punto común de conexión, que es el punto de la red de distribución al que se conectan las cargas o el consumidor (Ibáñez, Camiña, Torres, \& Bolaño, 2013).

Los valores instantáneos de las magnitudes eléctricas de un circuito o de un sistema eléctrico, como la tensión o la corriente, generalmente varían en el tiempo. Esta variación permite realizar su análisis y estudio mediante señales y sus correspondientes formas de onda.

Existen muchos tipos de señales alternas, pero a pesar de ello en el estudio de la electricidad, la denominación de señal alterna se suele restringir a un tipo especial de estas, que son las señales alternas sinusoidales.

El análisis de circuitos eléctricos en régimen sinusoidal, es decir, cuando las fuentes de excitación producen tensiones que varían en el tiempo de una forma periódica según una expresión sinusoidal, se realiza mediante fasores. (Ibáñez, Camiña, Torres, \& Bolaño, 2013).
A continuación, se observa una representación fasorial de ondas sinusoidales:

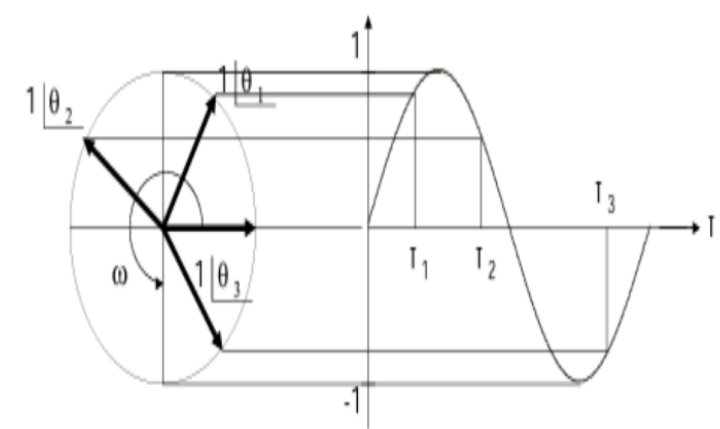

Figura 1 Representación fasorial de ondas sinusoidales Fuente. Alimentación de cargas críticas y calidad de la energía eléctrica. (Ibáñez, Camiña, Torres, \& Bolaño, 2013)

A principios del siglo XIX, el matemático y físico Jean-Baptiste Joseph Fourier demostró que cualquier función que sea periódica y continua por tramos se puede descomponer como la suma de una constante y de infinitos términos funcionales sinusoidales, llamados armónicos. Así, si una señal periódica $g(t)$ cumple las siguientes condiciones:

- $\quad$ Ser continua o tener un número finito de discontinuidades en un periodo.

Tener un numero finito de máximos y mínimos en un periodo.

Tener un valor medio finito.

Se puede descomponer como una serie suma de términos de senos y cosenos de la forma:

$g(t)=a_{0}+\sum_{k=1}^{\infty}\left\{b_{k} \cos (k \omega t)+c_{k} \operatorname{sen}(k \omega t)\right\}$

Donde $k$ es un numero entero y la pulsación $\omega$ es:

$\omega=2 \pi f=\frac{2 \pi}{T}$

Siendo $f$ y $T$ la frecuencia y el periodo de la señal $g(t)$.

A esta suma de términos se le denomina serie de Fourier. El coeficiente $a_{0}$ representa la componente continua de la onda, el termino $a_{1} \cos \left(\omega t+\varphi_{1}\right)$ se denomina componente fundamental y tiene la misma frecuencia de la señal $g(t)$, y a cada uno de los términos $a_{k} \cos \left(k \omega t+\varphi_{k}\right)$ restantes se le denomina armónico de orden $\mathrm{k}$ de la serie. 
En la siguiente figura se muestra la forma de onda, una vez suprimida la componente continua, considerado hasta distintos valores de $k$.

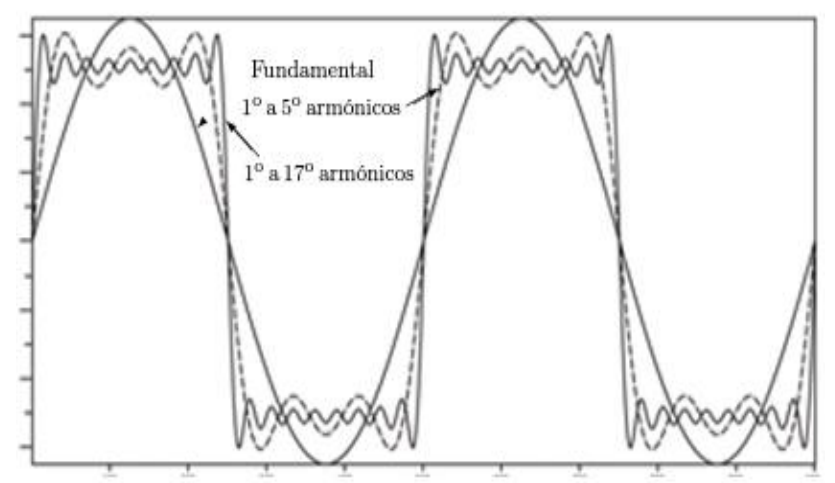

Figura 2 Onda fundamental y armónicos

Fuente: Alimentación de cargas críticas y calidad de la energía eléctrica. (Ibáñez, Camiña, Torres, \& Bolaño, 2013)

Como se puede observar, la onda fundamental representa la onda que proporciona el suministro eléctrico, ya sea a 50 o $60 \mathrm{~Hz}$, la onda punteada contiene la fundamental y los armónicos hasta el $5^{\circ}$ y finalmente se muestra el resultado de superponer la onda fundamental y sus armónicos hasta el orden $17^{\circ}$ (Ibáñez, Camiña, Torres, \& Bolaño, 2013).

En la actualidad, gracias al constante crecimiento y auge de la tecnología, en los sistemas eléctricos tanto de baja como de media tensión hay conectado un número cada vez mayor de cargas no lineales a la red, es decir, de cargas en las que la relación entre la intensidad que circula por ellas y la caída de tensión entre sus terminales no se puede representar mediante una función lineal.

Algunos ejemplos de cargas no lineales son:

Equipos de soldadura, hornos de arco y sistemas electrolíticos.

Convertidores electrónicos, como rectificadores, inversores y fuentes de alimentación.

Variadores de velocidad de máquinas eléctricas, tanto de A.C. como de D.C.

Equipos de informática, de oficina y electrodomésticos.

Los balastros electrónicos utilizados para iluminación con lámparas fluorescentes y con lámparas de descarga.
El problema con este tipo de cargas radica en que al aplicar una tensión sinusoidal a una carga no lineal la corriente que circula por ella es no sinusoidal. Además, muchas de ellas contienen sistemas de conmutación con componentes electrónicos de potencia que utilizan solo una parte de la onda de tensión y en consecuencia, demandan corriente solo en ciertos instantes, en forma de pulsos, lo que hace que la intensidad demandada e inyectada en la red este fuertemente distorsionada. Esas corrientes no sinusoidales, a su vez, al circular por las impedancias de la propia red de alimentación, hacen que las caídas de tensión en ellas sean también no sinusoidales. En consecuencia, todo este proceso da lugar a que tanto las tensiones como las corrientes que hay en el sistema eléctrico de distribución sean no sinusoidales, aunque la tensión de alimentación si lo sea.

Las cargas no solo se pueden ver afectadas por esa distorsión de las ondas de tensión y de intensidad, las cuales se denominan distorsión armónica, sino que en muchos casos también el origen de ella. El estudio de la distorsión armónica se distingue entre la distorsión de tensión asociada generalmente al sistema de alimentación de energía eléctrica, y la distorsión de corriente, asociada a las cargas no lineales que hay conectadas en él (Ibáñez, Camiña, Torres, \& Bolaño, 2013).

A continuación, se muestra una tabla con los valores de la frecuencia, empezando por la fundamental que es de $60 \mathrm{~Hz}$, hasta el $9^{\circ}$ armónico, mostrando la amplitud relativa y secuencia para cada caso, la cual se determina considerando negativo cuando el armónico es del orden $(3 n-1)$, positiva cuando es $(3 n+1)$ y 0 cuando es del orden $(3 n)$.

\begin{tabular}{|c|c|c|c|}
\hline $\begin{array}{l}\text { Orden del } \\
\text { armónico }\end{array}$ & Frecuencia & secuencia & $\begin{array}{l}\text { Amplitud } \\
\text { relativa }\end{array}$ \\
\hline Fundamental & $60 \mathrm{~Hz}$ & + & $100 \%$ \\
\hline $3^{\circ}$ & $180 \mathrm{~Hz}$ & 0 & $33 \%$ \\
\hline $5^{\circ}$ & $300 \mathrm{~Hz}$ & - & $20 \%$ \\
\hline $7^{\circ}$ & $420 \mathrm{~Hz}$ & + & $14 \%$ \\
\hline $9^{\circ}$ & $540 \mathrm{~Hz}$ & 0 & $11 \%$ \\
\hline Orden n & $60 \mathrm{n} \mathrm{Hz}$ & $\ldots$ & $100 / \mathrm{n} \%$ \\
\hline
\end{tabular}

Tabla 1 Contenido armónico para una frecuencia fundamental de $60 \mathrm{~Hz}$

Fuente. Basada de las notas de aplicación de Power Electronics “Armónicos en sectores industriales" (Sales, Chilet, \& Grau, 2011) 
Este fenómeno al estar siempre presente en las redes eléctricas de distribución y transporte de energía eléctrica afectan de forma permanente a la propia red y a las cargas conectadas a ella.

El efecto de los armónicos sobre los equipos depende de la naturaleza de estos, así como de la magnitud, frecuencia y fase de estos. Algunos de los efectos directos más importantes que producen son:

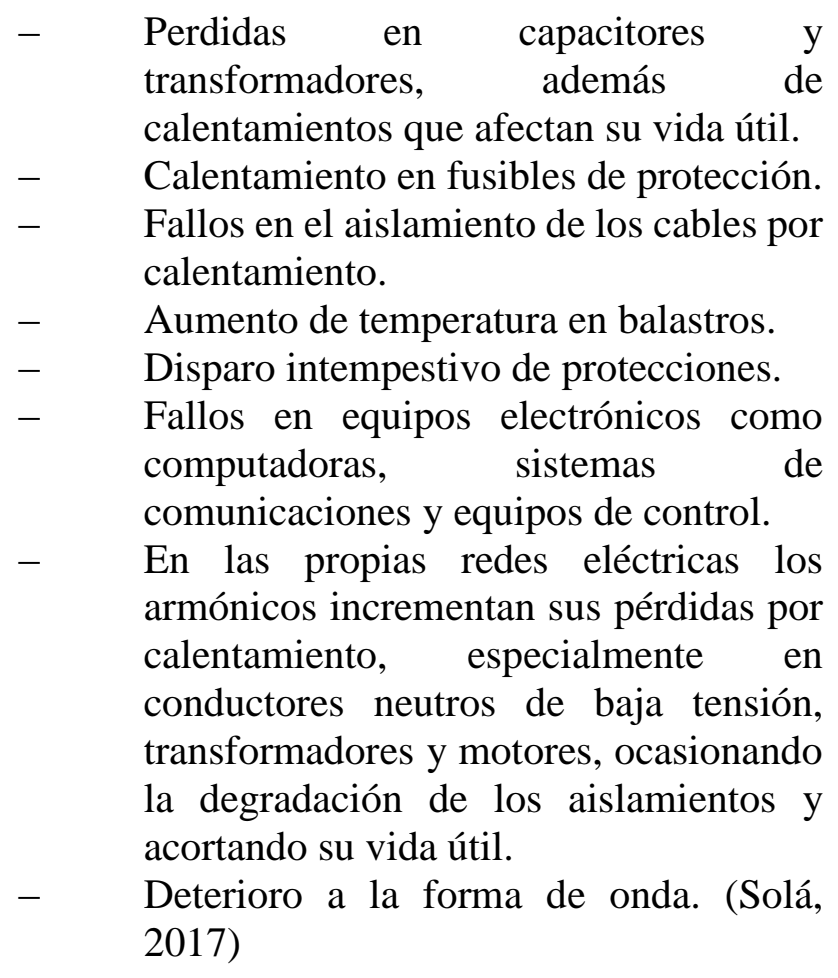

Las energías alternativas como los sistemas fotovoltaicos producen corriente continua mientras que las cargas que se utilizan en la industria y viviendas son de corriente alterna, por lo que es necesario un convertidor de potencia que permita la conversión de C.D a C.A, o como mejor se le conoce, un inversor.

Los sistemas fotovoltaicos interconectados a la red (SFVI) son equipos que aprovechan la radiación solar, la convierten en electricidad y continúa interactuando con la red eléctrica.

Hay tres principales componentes de un SFVI, los cuales son los siguientes:

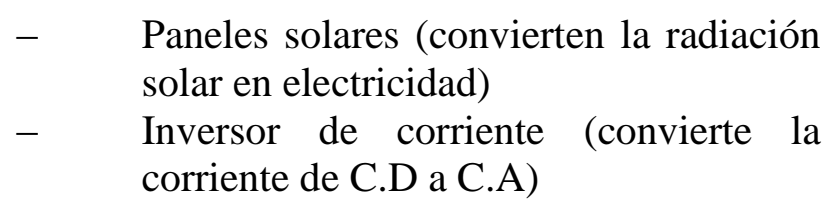

Medidor bidireccional (mide cuanta electricidad se consume de la red eléctrica y "descuenta" la electricidad que se entrega a la red)

A continuación, se muestra un diagrama de cómo está constituido un SFVI (ENERCEN, 2013):

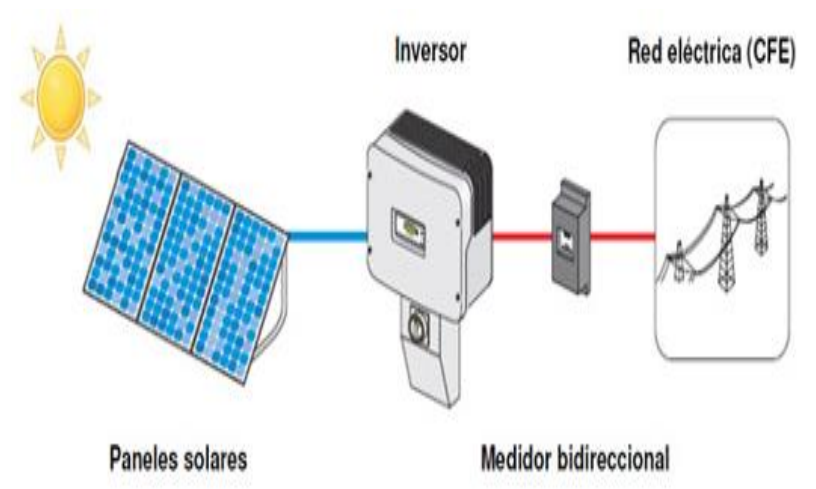

Figura 3 Diagrama de un SFVI.

Fuente. ENERCEN

El inversor después de convertir la corriente eléctrica para que esta sea compatible con la red eléctrica, este la inyecta a la red con la calidad que genera.

El número de inversores conectados a la red eléctrica está en aumento, comportándose como cargas no lineales, esto gracias a que estos aparatos constan de una etapa de potencia realizada con transistores, que trocean la corriente C.D alterándola y creando una forma de onda casi sinusoidal. La salida puede ser monofásica o trifásica dependiendo de las características y potencia de la carga.

Por lo tanto, al estar constituidos por elementos de electrónica de potencia, consumen corrientes no sinusoidales, produciendo una distorsión de la tensión de la red eléctrica, provocando un consumo de potencia reactiva y de distorsión que degrada el rendimiento de las centrales energéticas y de sus líneas de distribución (Sales, Chilet, \& Grau, 2011).

Las señales de tensión y corriente generadas por los inversores presentan componentes armónicas y dado a que este fenómeno no puede erradicarse en su totalidad, si puede haber soluciones para reducir la distorsión armónica, un ejemplo es el uso de filtros pasivos o activos. 
Esto es, después de aplicar una adecuada técnica de modulación, se añade al sistema un filtro pasivo a la salida que permita el paso de la componente fundamental e impida el paso a las restantes componentes armónicas.

En la siguiente figura se aprecia un diagrama para reducir los armónicos generados por un inversor (Sales, Chilet, \& Grau, 2011).

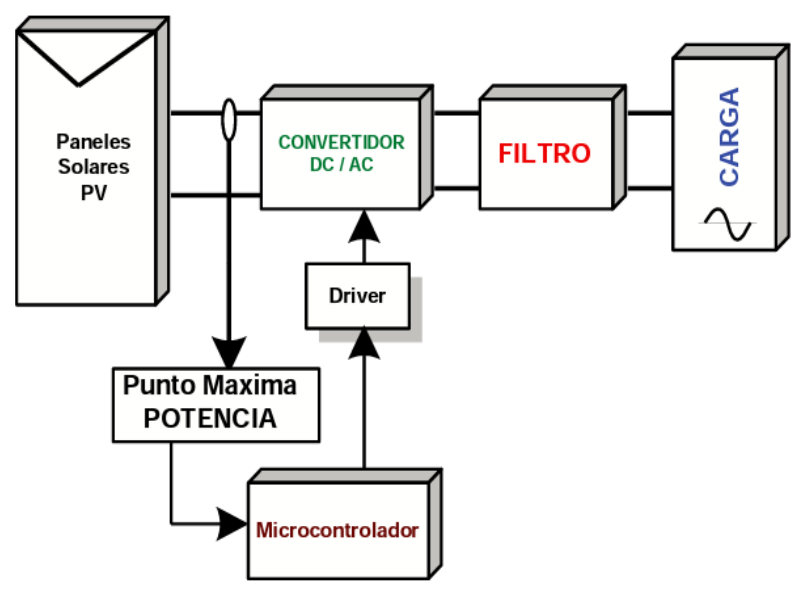

Figura 4 Diagrama de un sistema fotovoltaico Con filtro pasivo

Fuente. Convertidores electrónicos: energía solar fotovoltaica. Aplicaciones y diseño

\section{Hipótesis}

Los SFVI aportan armónicos a la red por lo que la calidad de la energía que suministra la red se afectará en la medida en que cada vez más sistemas fotovoltaicos se interconecten a la misma, como es el objetivo respecto a las energías renovables.

\section{Metodología}

Para determinar el grado de distorsión armónica que generan los inversores de los SFVI a la red, fue necesario realizar mediciones y pruebas a 4 diferentes inversores, 2 de ellos de modelos y fabricantes diferentes y los otros configurados en el laboratorio de energías renovables de la UTS.

Para las mediciones y análisis a los inversores se utilizó un analizador de energía calibrado, HT Instruments PQA 824 el cual se muestra a continuación:

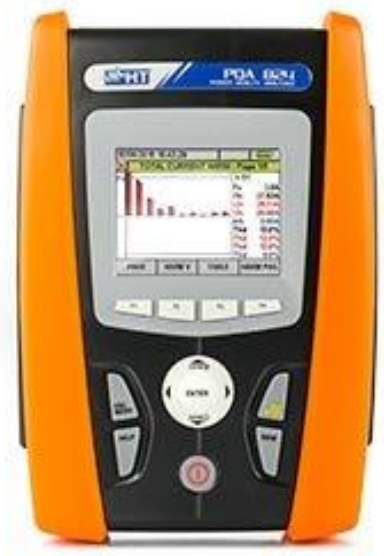

Figura 5 Analizador de energía PQA 824 Fuente: Elaboración Propia

El PQA 824 es un analizador de red trifásico y monofásico, este permite la visualización de los parámetros en las modalidades numérica y gráfica, tanto para el análisis periódico como para el análisis armónico. Entre sus principales funciones están:

- $\quad$ Medición de voltaje CA/CD en sistemas monofásico/trifásico Max $1000 \mathrm{~V}$

- Corriente CA/CD en sistemas monofásico/trifásico Max $3000 \mathrm{~A}$

Factor de potencia

- $\quad$ Armónicos de tensión y corriente hasta el $64^{\circ}$ con THD\%

- $\quad$ Anomalías de tensión (huecos, picos)

- $\quad$ Flickers

- $\quad$ Corriente de neutro

- $\quad$ Formas de onda de tensión y corriente

- Tablas o histogramas de armónicos y

THD\% (HT-Instruments, 2019)

Con este analizador se compara el nivel de THD (distorsión armónica total) generada por los diferentes inversores bajo estudio y plantear una solución que disminuya este fenómeno.

La especificación CFE L0000-45 permite saber las desviaciones permisibles en las formas de onda de tensión y corriente en el suministro y consumo de energía eléctrica. Según esta especificación, el contenido armónico de la tensión en el punto de acometida para formas de onda periódica debe limitarse a los niveles que marca la siguiente tabla: 


\begin{tabular}{|c|c|c|}
\hline Tensión kV & $\begin{array}{l}\text { Componente } \\
\text { Armónico } \\
\text { Individual } \\
\text { Máximo de } \\
\text { Tensión } \\
\text { (CAIMT) }\end{array}$ & $\begin{array}{l}\text { Distorsión } \\
\text { Armónica } \\
\text { Total de } \\
\text { Tensión } \\
\text { (DATT) \% }\end{array}$ \\
\hline Menor de 1 & 6 & 8 \\
\hline De 1 a 35 & 5 & 6.5 \\
\hline Mayor de 35 & 2 & 3 \\
\hline
\end{tabular}

Tabla 2 Límites máximos de CAIMT y DATT Fuente: Especificación CFE LOO00-45

Además, los consumidores deben limitar el contenido armónico que generan a la corriente que demandan según su impedancia relativa y su nivel de tensión, de acuerdo con la siguiente tabla.

\begin{tabular}{|c|c|}
\hline $\begin{array}{l}\text { Impedancia relativa } \\
\qquad\left(\mathrm{I}_{\mathrm{cc}} / \mathrm{IL}\right)\end{array}$ & $\begin{array}{l}\text { Distorsión Armónica } \\
\text { Total de Demanda } \\
\text { (DATD) \% }\end{array}$ \\
\hline$\left(\mathrm{I}_{\mathrm{cc}} / \mathrm{IL}\right)<20$ & 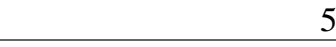 \\
\hline $20 \leq\left(\mathrm{I}_{\mathrm{cc}} / \mathrm{IL}\right)<50$ & 8 \\
\hline $50 \leq\left(\mathrm{I}_{\mathrm{cc}} / \mathrm{IL}\right)<100$ & 12 \\
\hline $100 \leq\left(\mathrm{I}_{\mathrm{cc}} / \mathrm{IL}\right)<1000$ & 15 \\
\hline$\left(\mathrm{I}_{\mathrm{cc}} / \mathrm{IL}\right) \geq 1000$ & 20 \\
\hline
\end{tabular}

Tabla 3 Distorsión armónica máxima permitida en corriente para baja, media y alta tensión hasta $69 \mathrm{kV}$ Fuente: Especificación CFE L0000-45

\section{Pruebas a inversores}

\section{SUNFORCE 1000-Watt}

Potencia de salida 1000 Watts, especificaciones:

\begin{tabular}{|c|c|}
\hline \multirow{2}{*}{\multicolumn{2}{|c|}{ Especificaciones }} \\
\hline & \\
\hline \multicolumn{2}{|l|}{ Potencia de salida } \\
\hline Continua & $1000 \mathrm{~W}$ \\
\hline De arranque & $2000 \mathrm{~W}$ \\
\hline \multicolumn{2}{|c|}{ Entrada de CC a la batería } \\
\hline Sistema $12 \mathrm{~V} \mathrm{CC}$ & $\begin{array}{l}12 \mathrm{~V} \mathrm{CC} \text { nominal }(11-15 \mathrm{~V}) \\
\text { operativo }\end{array}$ \\
\hline \multicolumn{2}{|c|}{ Inverter ac output } \\
\hline Tensión & 110V CA RMS +/- $10 \%$ \\
\hline Frecuencia & $60 \mathrm{~Hz}+/-2 \%$ \\
\hline Forma de onda & Onda sinusoidal pura \\
\hline Eficiencia & $90 \%$ \\
\hline $\begin{array}{ll}\text { Distorsión } & \text { Total } \\
\text { Armonizada } & \\
\end{array}$ & $4 \%+/-1 \%$ \\
\hline $\begin{array}{l}\text { Variación de la Tensión } \\
\text { de Salida }\end{array}$ & $110 \mathrm{VAC}+/-10 \%$ \\
\hline Variación de Frecuencia & $60 \mathrm{~Hz}+/-2 \%$ \\
\hline Total KVA (+/- 10\%) & 1.4 \\
\hline
\end{tabular}

Tabla 4 Especificaciones del inversor SUNFORCE Fuente. SUNFORCE Products
A este inversor se le realizo una prueba sin carga o "en vacío" para determinar el grado de THD que genera, a continuación, se muestran las lecturas obtenidas:

\section{Sin carga}

En la figura 6, se muestra la forma de onda del voltaje a la salida del inversor con un alto grado de distorsión.

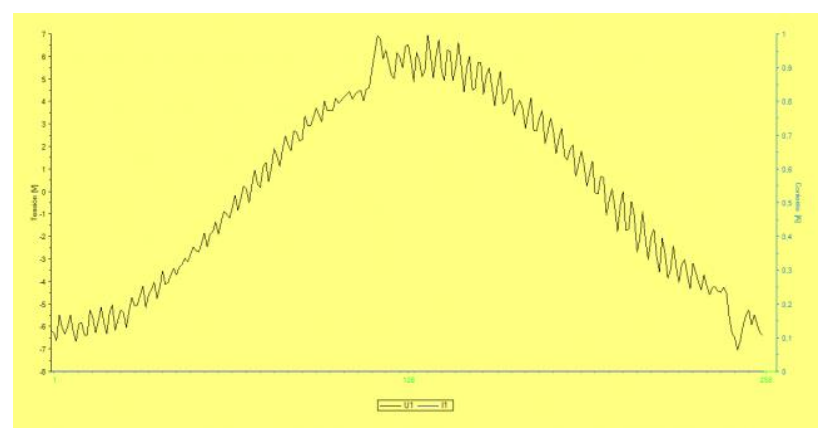

Figura 6 Forma de onda de voltaje del inversor sin carga Fuente: Elaboración Propia

En la figura 7 se puede apreciar el espectro de frecuencias que genera el inversor al trabajar sin carga conectada, aquí se ve el grado de distorsión que se genera y hasta el orden $64^{\circ}$

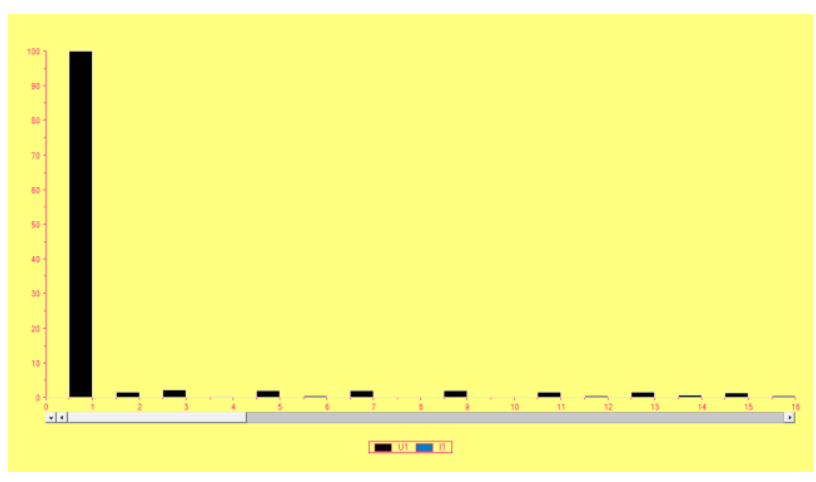

Figura 7 Espectro de frecuencias para inversor sin carga Fuente: Elaboración Propia

En la figura 8 se puede apreciar en el multímetro el grado de THD para cuando el inversor está trabajando sin carga, en el cual se muestra un THDv de $7.13 \%$ y un $\mathrm{THD}_{\mathrm{I}}$ de $0 \%$. 


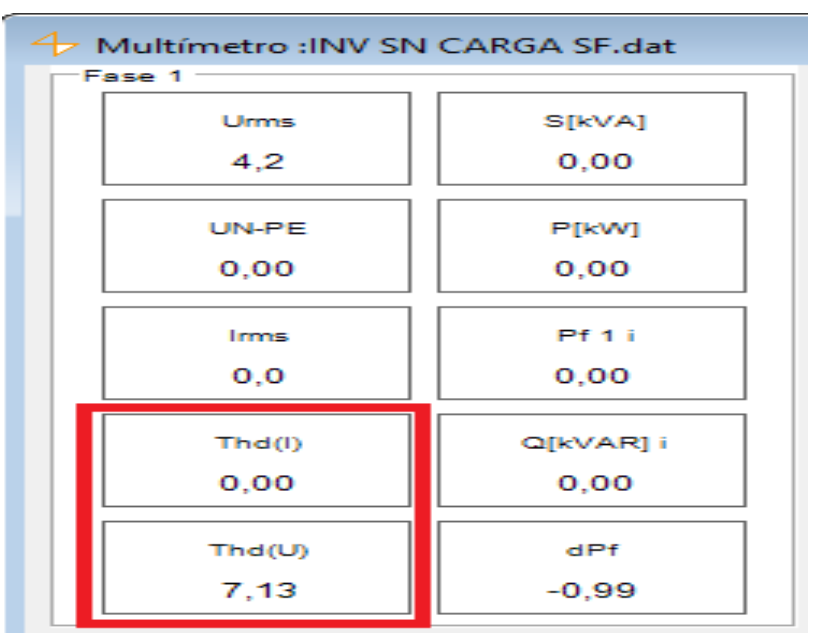

Figura 8 Valores de THD para inversor sin carga Fuente: Elaboración Propia

Además, a dicho inversor se le realizaron las pruebas trabajando con las siguientes cargas conectadas:

\section{- Carga resistiva \\ - Carga resistiva - inductiva - capacitiva \\ - Carga no lineal}

Las primeras pruebas se realizaron conectando una carga a la vez, a continuación, se muestran las lecturas obtenidas por el analizador de energía donde se ve el grado de THD tanto para voltaje como para corriente.

\section{Prueba a carga resistive}

Como se puede ver a en la figura 9 , se muestra la forma de onda tanto de voltaje (color negro) como de corriente (color azul) a la salida del inversor, en la cual se puede apreciar el aporte de distorsión en cada una de ellas siendo la onda de corriente la más afectada ya que es la que presenta mayor distorsión.

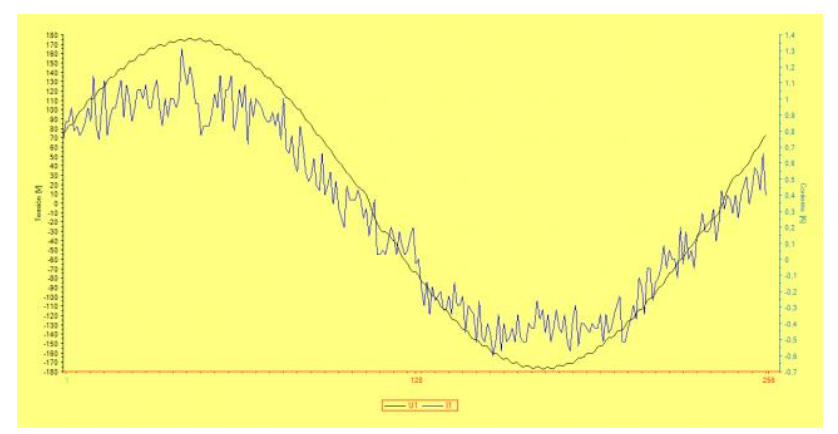

Figura 9 Forma de onda de voltaje y corriente del inversor con carga resistiva.

Fuente: Elaboración Propia
En la figura 10 se observa el espectro de frecuencias en el cual se aprecia el THD y el número de armónicos presentes, para este caso hasta el $63^{\circ}$ armónico.

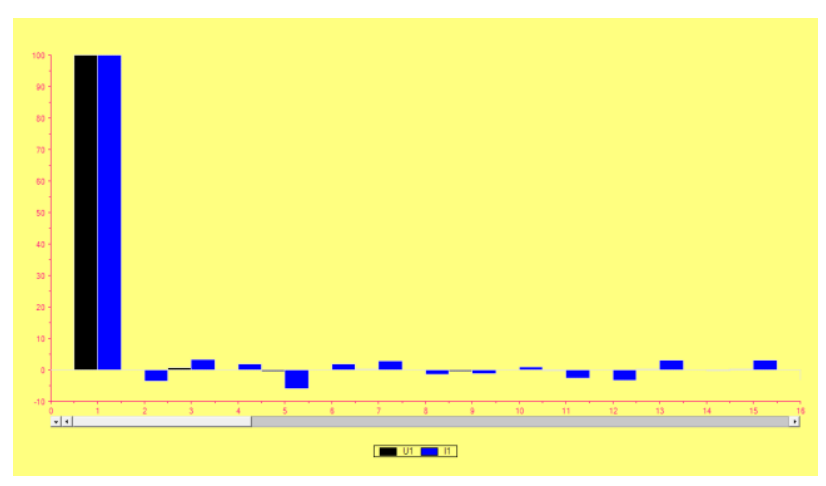

Figura 10 Espectro de frecuencias para inversor con carga resistiva

Fuente. Elaboración Propia

Por último, se observa en el multímetro el grado de THD para voltaje y corriente que aporta el inversor al estar trabajando con esta carga conectada, donde el THDV es de $1.52 \%$ y el THD I es igual a $13.81 \%$.

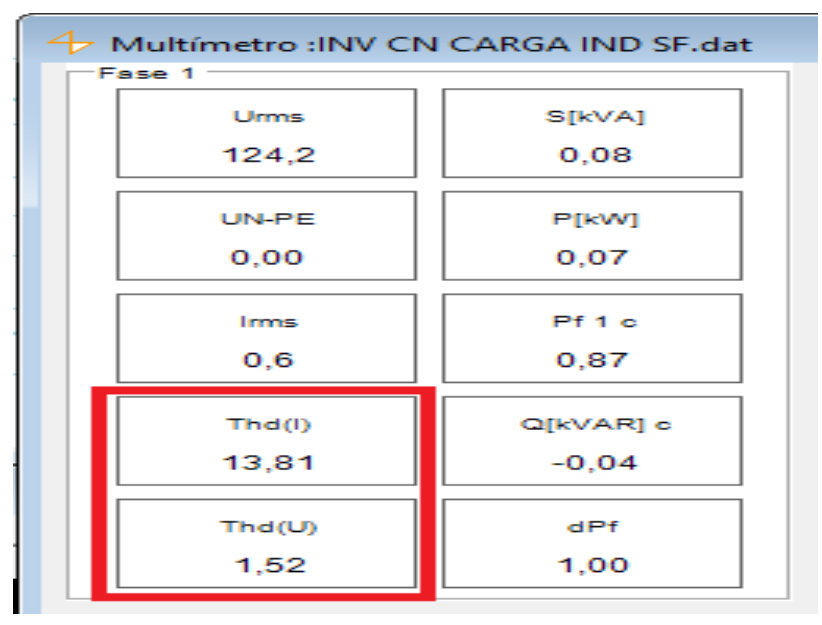

Figura 11 Valores de THD para inversor con carga resistiva

Fuente. Elaboración Propia

\section{Carga capacitiva/inductiva}

Como se puede ver a continuación en la figura 12 , se muestra la forma de onda de voltaje a la salida del inversor, en la cual se aprecia cierto grado de distorsión en dicha onda. 


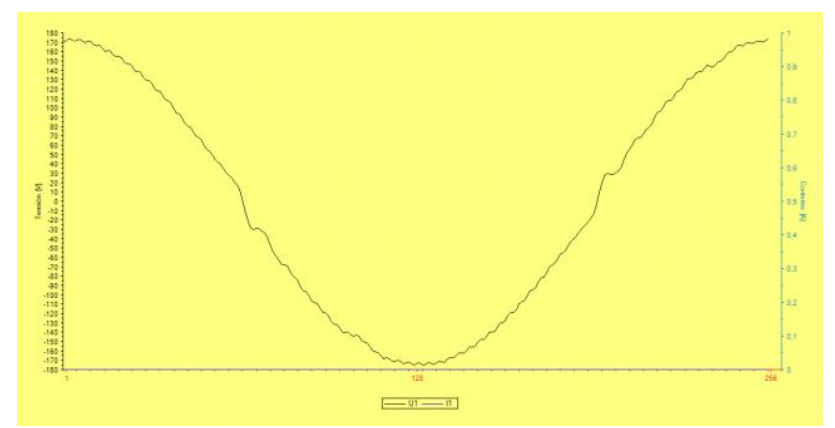

Figura 12 Forma de onda de voltaje del inversor con carga capacitiva/inductiva

Fuente. Elaboración Propia

Enseguida en la figura 13 se muestra el espectro de frecuencias en el cual se aprecia el THD y el número de armónicos presentes, para este caso hasta el $35^{\circ}$ armónico.

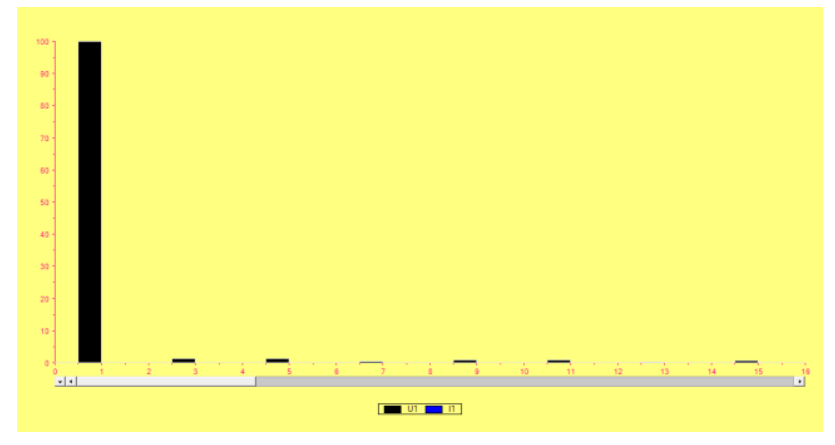

Figura 13 Espectro de frecuencias para inversor con carga capacitiva/inductiva.

Fuente. Elaboración Propia

Por último, se observa en el multímetro el grado de THD para voltaje que aporta el inversor al estar trabajando con esta carga conectada, donde el THDv es de $2.95 \%$ y el THDI es igual a $0 \%$.

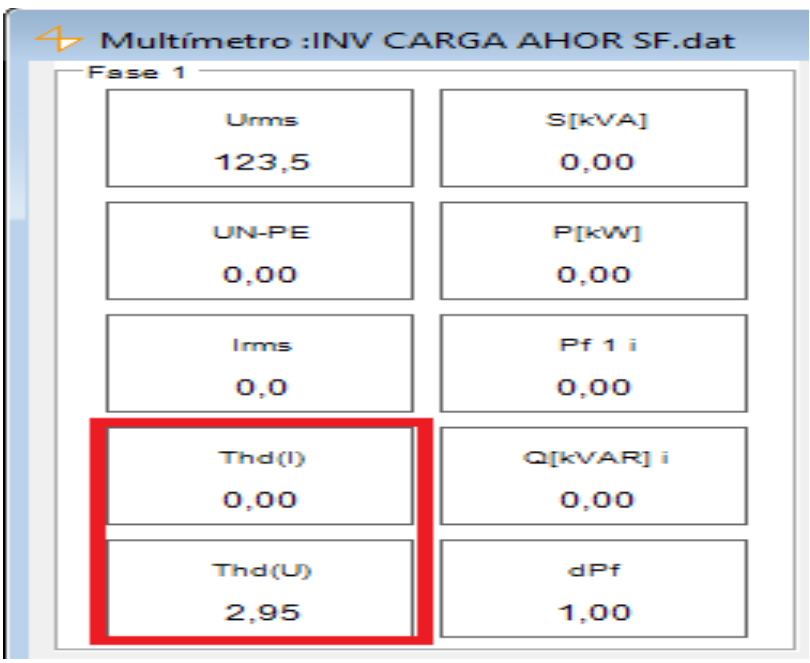

Figura 14 Valores de THD para inversor con carga capacitiva/inductive

Fuente: Elaboración Propia

\section{Carga no lineal}

Como se ve en la figura 15, la forma de onda de voltaje a la salida del inversor, en la cual se aprecia cierto grado de distorsión en dicha onda.

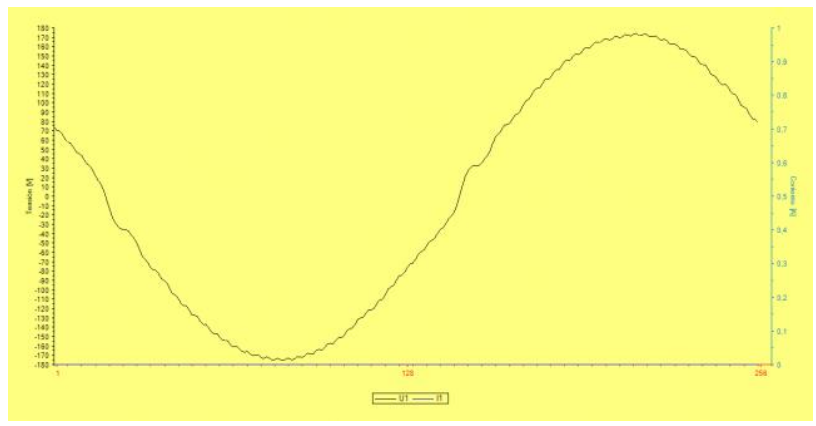

Figura 15 Forma de onda de voltaje del inversor con carga no lineal

Fuente: Elaboración Propia

Enseguida en la figura 16 se muestra el espectro de frecuencias en el cual se aprecia el THD y el número de armónicos presentes, para este caso hasta el $29^{\circ}$ armónico.

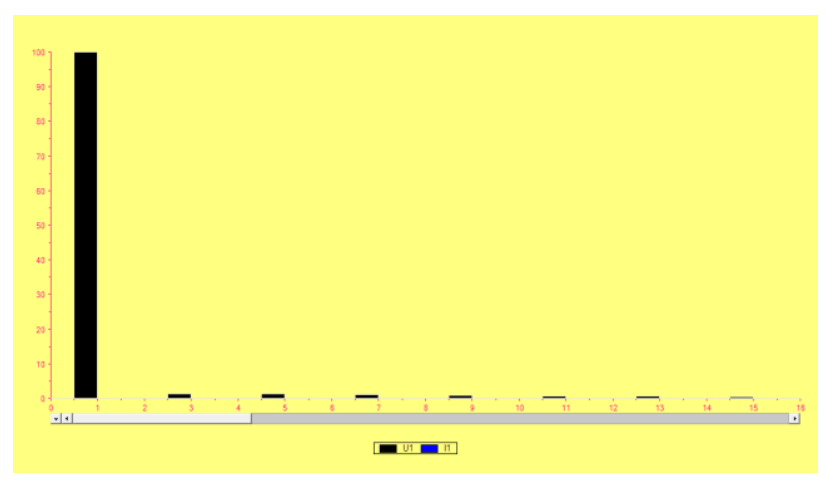

Figura 16 Espectro de frecuencias para inversor con carga no lineal

Fuente: Elaboración Propia

Por último, se observa en el multímetro el grado de THD para voltaje que aporta el inversor al estar trabajando con esta carga conectada, donde el $\mathrm{THD}_{\mathrm{V}}$ es de $2.92 \%$ y el $\mathrm{THD}_{\text {I }}$ es igual a $0 \%$. 


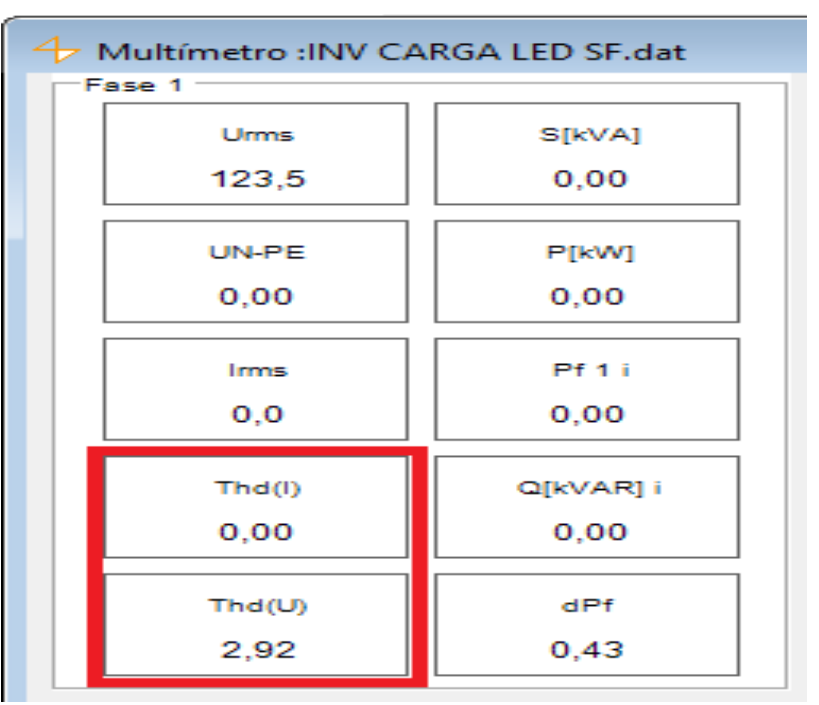

Figura 17 Valores de THD para inversor con carga no lineal

Fuente: Elaboración Propia

Una vez realizadas las pruebas al inversor conectando una carga a la vez, se realizaron combinaciones para ver su efecto, las combinaciones fueron las siguientes:

Resistiva - no lineal

Resistiva - capacitiva/inductiva

No lineal - capacitiva/inductiva

A continuación, se muestran las lecturas obtenidas por el analizador de energía donde se ve el grado de THD tanto para voltaje como para corriente.

\section{Resistiva - no lineal}

En la figura 18, se muestra la forma de onda tanto de voltaje (color negro) como de corriente (color azul) a la salida del inversor, en la cual se aprecia cierto grado de distorsión en cada una de ellas siendo la onda de corriente la más afectada ya que es la que presenta mayor distorsión.

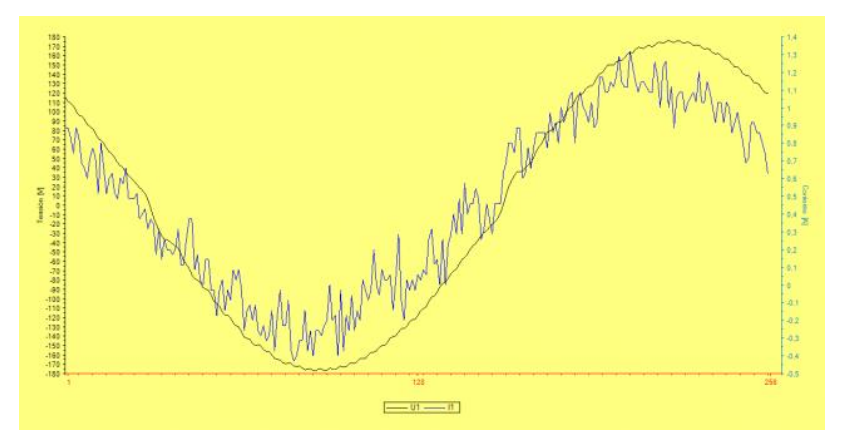

Figura 18 Forma de onda de voltaje y corriente del inversor con carga resistiva - no lineal.

Fuente: Elaboración Propia
En la figura 19 se muestra el espectro de frecuencias en el cual se aprecia el THD y el número de armónicos presentes, para este caso hasta el $63^{\circ}$ armónico.

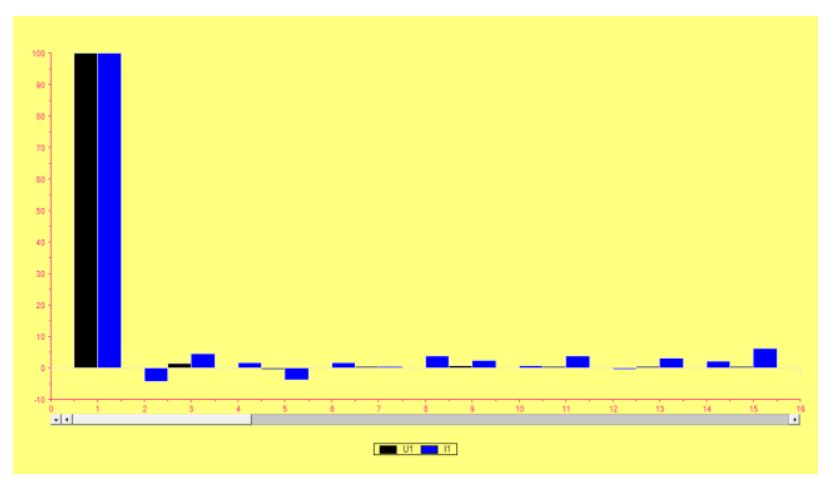

Figura 19 Espectro de frecuencias para inversor con carga resistiva - no lineal.

Fuente: Elaboración Propia

Por último, se puede observar en el multímetro el grado de THD para voltaje y corriente que aporta el inversor al estar trabajando con esta carga combinada, donde el THDv es de $2.40 \%$ y el THDI es igual a $15.05 \%$.

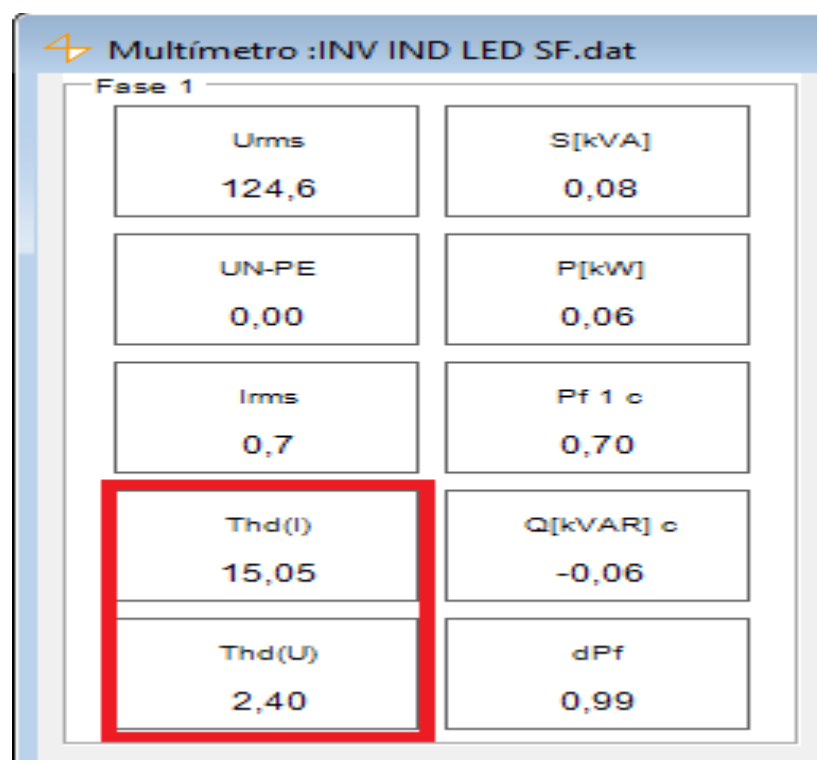

Figura 20 Valores de THD para inversor con carga resistiva - no lineal

Fuente: Elaboración Propia

\section{- $\quad$ Resistiva - capacitiva/inductiva}

A continuación, en la figura 21 , se muestra la forma de onda tanto de voltaje (color negro) como de corriente (color azul) a la salida del inversor, en la cual se aprecia cierto grado de distorsión en cada una de ellas siendo la onda de corriente la más afectada ya que es la que presenta mayor distorsión. 


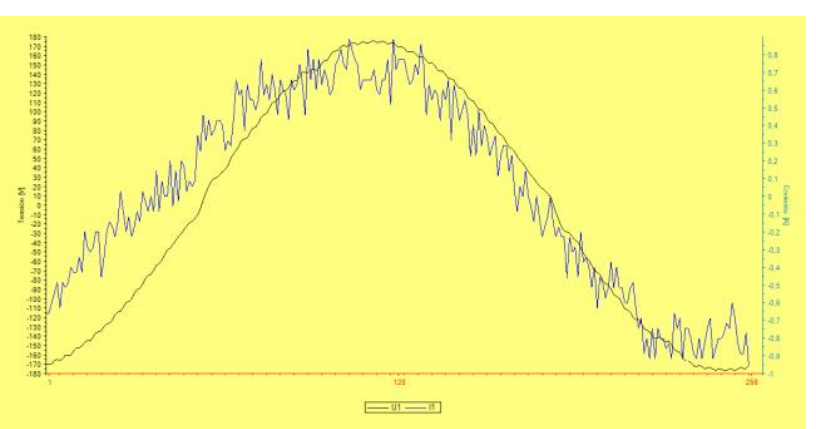

Figura 21 Forma de onda de voltaje y corriente del inversor con carga resistiva - capacitiva/inductiva Fuente. Elaboración Propia

En la figura 22 se muestra el espectro de frecuencias en el cual se puede apreciar el THD y el número de armónicos presentes, para este caso hasta el $63^{\circ}$ armónico.

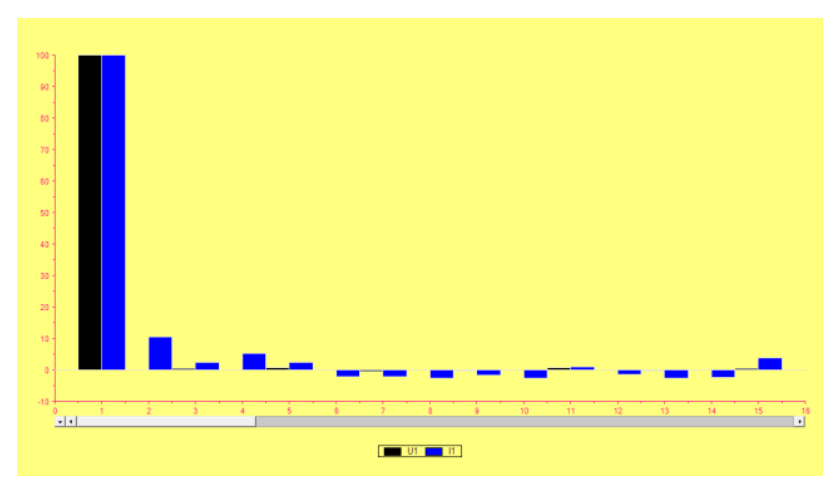

Figura 22 Espectro de frecuencias para inversor con carga resistiva - capacitiva/inductiva

Fuente. Elaboración Propia

Por último, se observa en el multímetro el grado de THD para voltaje y corriente que aporta el inversor al estar trabajando con esta carga combinada, donde el THDv es de $1.77 \%$ y el THD I es igual a $16.48 \%$.

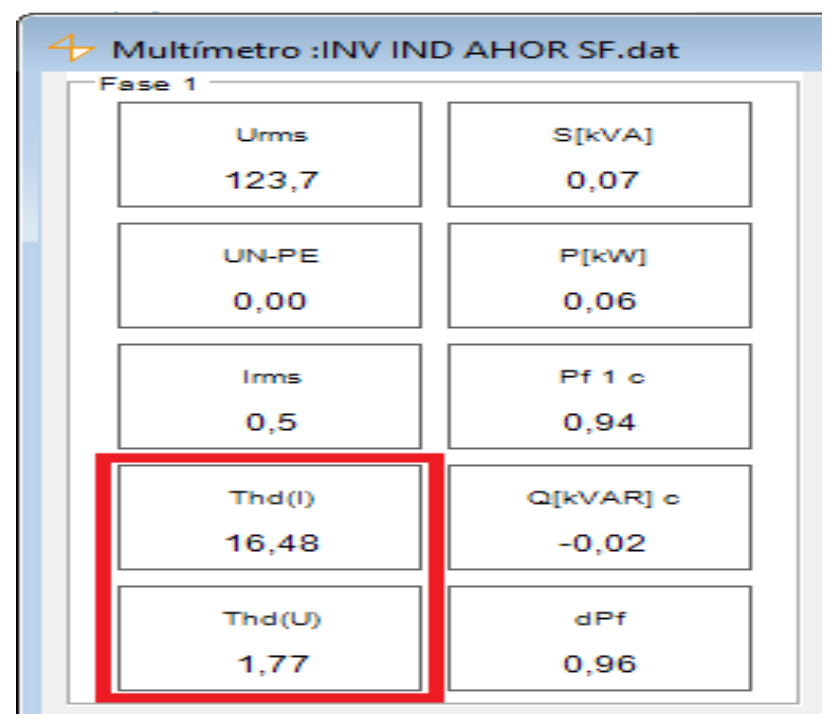

Figura 23 Valores de THD para inversor con carga resistiva - capacitiva/inductive

Fuente: Elaboración Propia

ISSN: 2523-2517

ECORFAN® Todos los derechos reservados

\section{- $\quad$ No lineal - capacitiva/inductiva}

En la figura 24, se muestra la forma de onda tanto de voltaje (color negro) como de corriente (color azul) a la salida del inversor, en la cual se puede apreciar cierto grado de distorsión en cada una de ellas siendo la onda de corriente la más afectada ya que es la que presenta mayor distorsión.

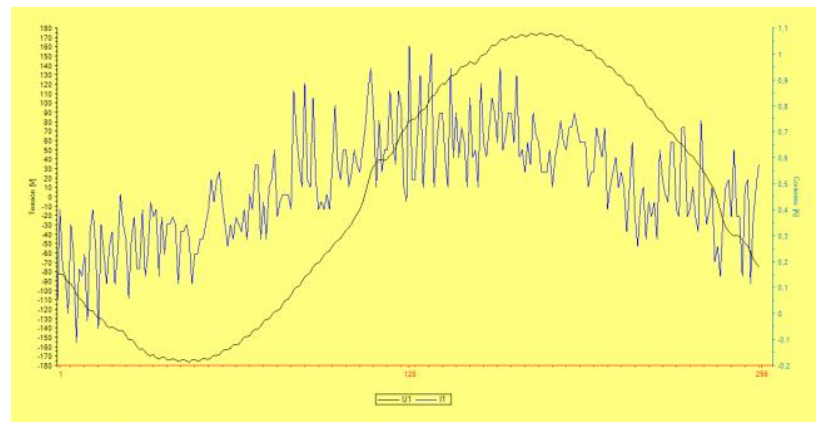

Figura 24 Forma de onda de voltaje y corriente del inversor con carga no lineal - capacitiva/inductiva Fuente: Elaboración Propia

En la figura 25 se muestra el espectro de frecuencias en el cual se aprecia el THD y el número de armónicos presentes, para este caso hasta el $63^{\circ}$ armónico.

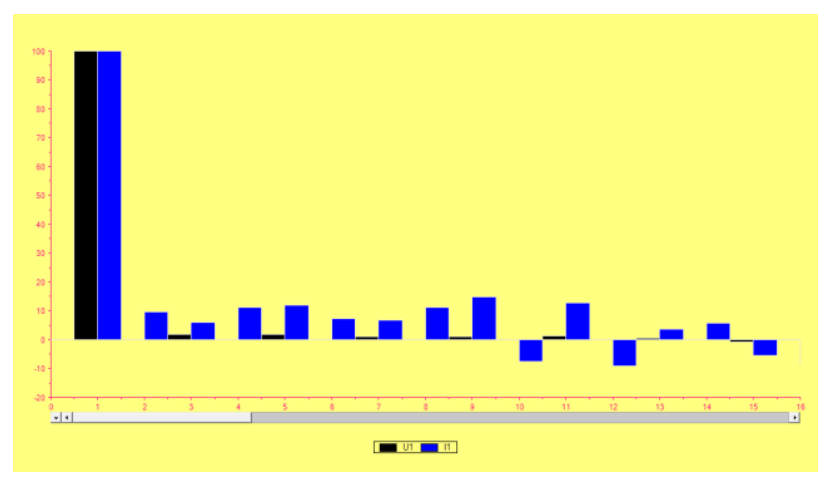

Figura 25 Espectro de frecuencias para inversor con carga no lineal - capacitiva/inductiva

Fuente: Elaboración Propia

Por último, se puede observar en el multímetro el grado de THD para voltaje y corriente que aporta el inversor al estar trabajando con esta carga combinada, donde el THD es de $3.71 \%$ y el THD I es igual a $48.90 \%$. 


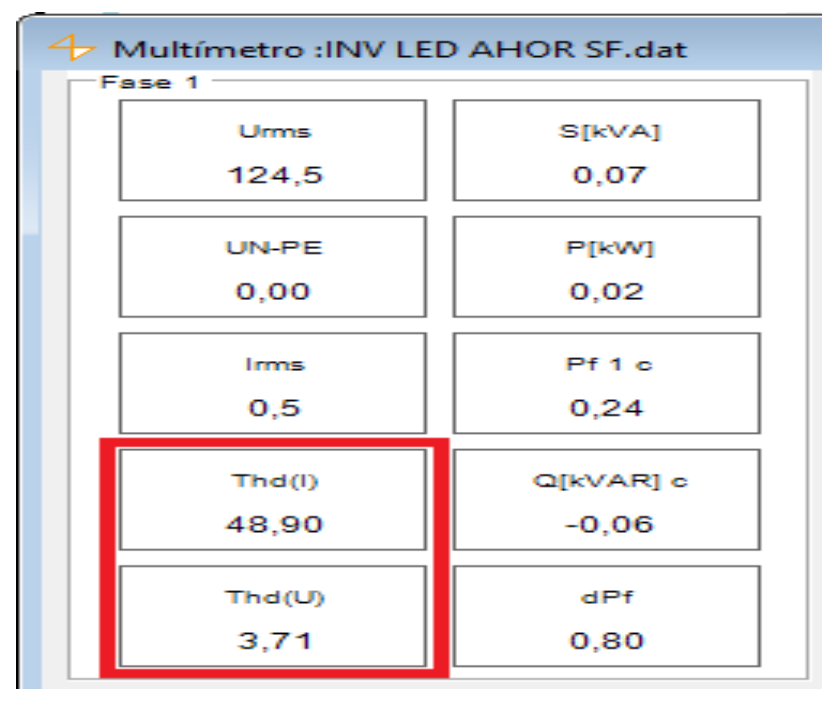

Figura 26 Valores de THD para inversor con carga no lineal - capacitiva/inductive

Fuente: Elaboración Propia

\section{Inversor trifásico configurado en el laboratorio}

Este inversor tiene la característica que puede comportarse también como rectificador, esto gracias a que está compuesto de tiristores, los cual al variar su ángulo de disparo provoca que este se comporte ya sea como uno u otro.

Para realizar la prueba fue necesario variar el ángulo de disparo de los tiristores para identificar en que ángulo este pasaba de rectificador a inversor, empezando desde su máximo que son $179.3^{\circ}$ hasta el mínimo $0^{\circ}$, en la figura 27 se puede apreciar el ángulo de disparo a su máximo que son $179.3^{\circ}$

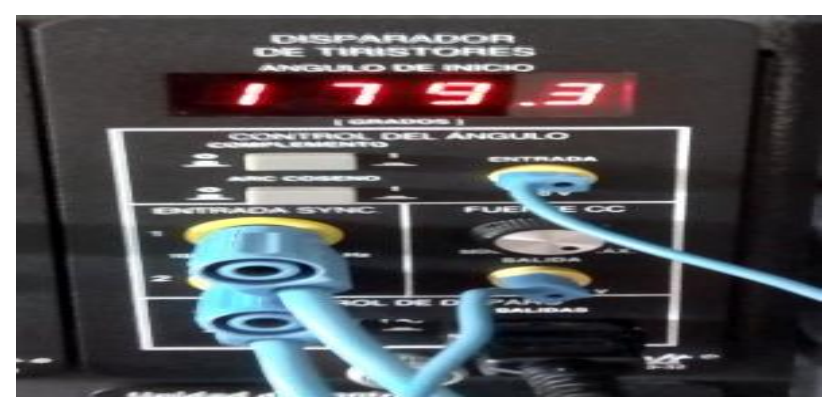

Figura 27 Angulo de disparo al máximo a 179.3ำ Fuente: Elaboración Propia

Al estar a este ángulo, el aparato se comporta como rectificador comportándose como se muestra en la figura 28.
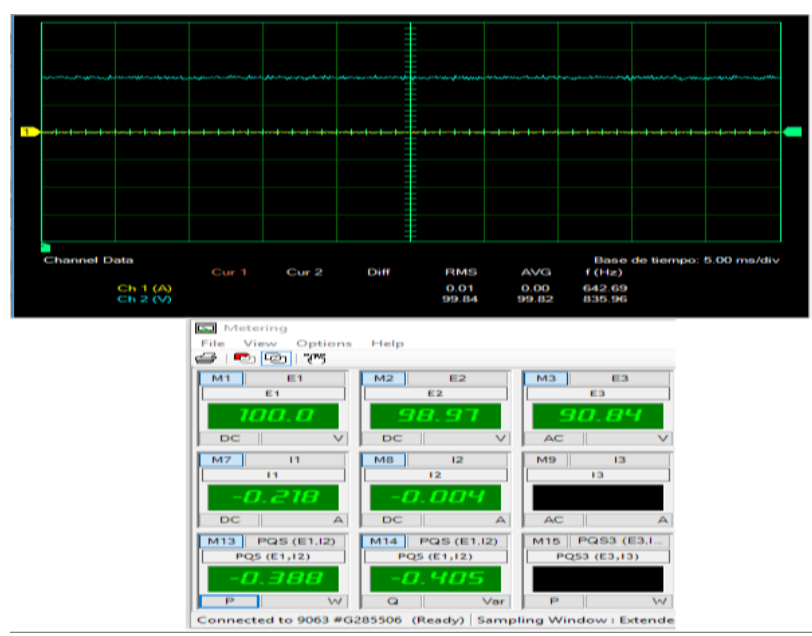

Figura 28 Gráfico y parámetros a $179.3^{\circ}$ Fuente: Elaboración Propia

A partir de ahí se fue modificando el ángulo disminuyéndolo poco a poco, no fue hasta que el ángulo llego a 53. $4^{\circ}$ que se observaron cambios, tanto en la forma de onda como en la potencia, en la figura 29 se aprecia el ángulo y en la figura 30 se pueden observar los cambios.

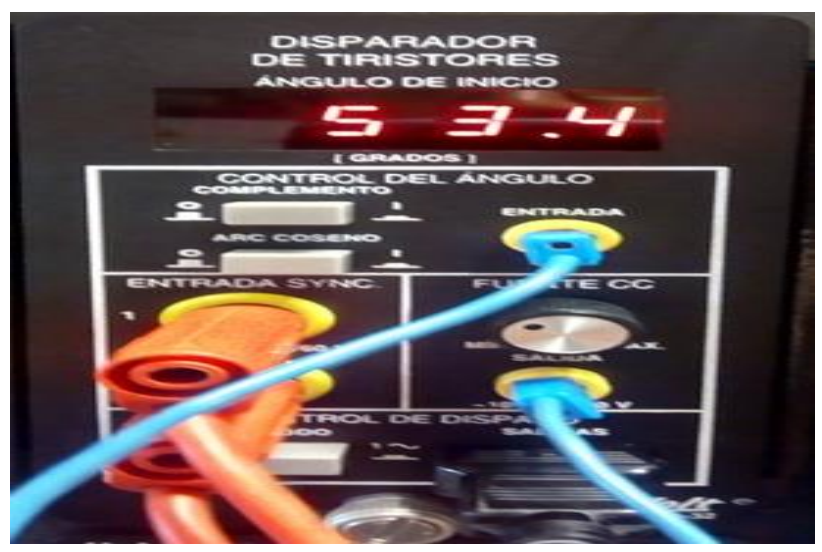

Figura 29 Angulo de disparo a 53. $4^{\circ}$ Fuente: Elaboración Propia
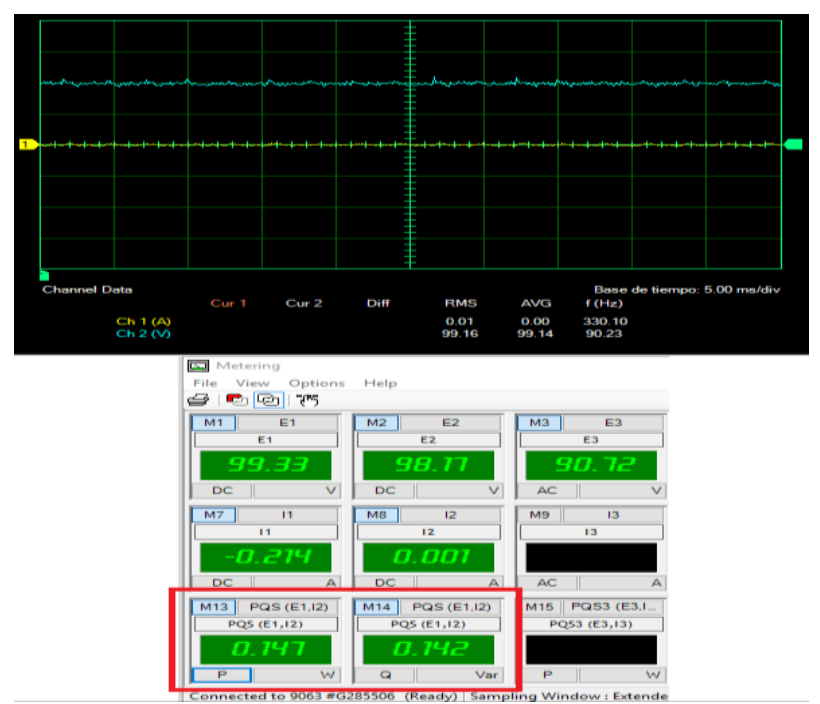

Figura 30 Gráfico y parámetros a $53.4^{\circ}$ Fuente: Elaboración Propia

DELGADO-PONCE, Luis, VARGAS-HERNÁNDEZ, Oscar Joel, MARTÍNEZ-MAGDALENO, Arturo y MENÉNDEZ-BENAVENTE, Luis Martín. Los armónicos que aportan los sistemas fotovoltaicos interconectados a la red un problema latente. Revista de Ingeniería Eléctrica. 2019. 
Aunque los cambios son mínimos en este punto, se puede ver que cumple con su función y que al ir disminuyendo su ángulo este se comporta como inversor, en la siguiente figura 31 se aprecia que el ángulo de disparo es de $15^{\circ}$ y en la figura 32 se pueden apreciar mejor los cambios en la forma de onda y un aumento de la potencia.

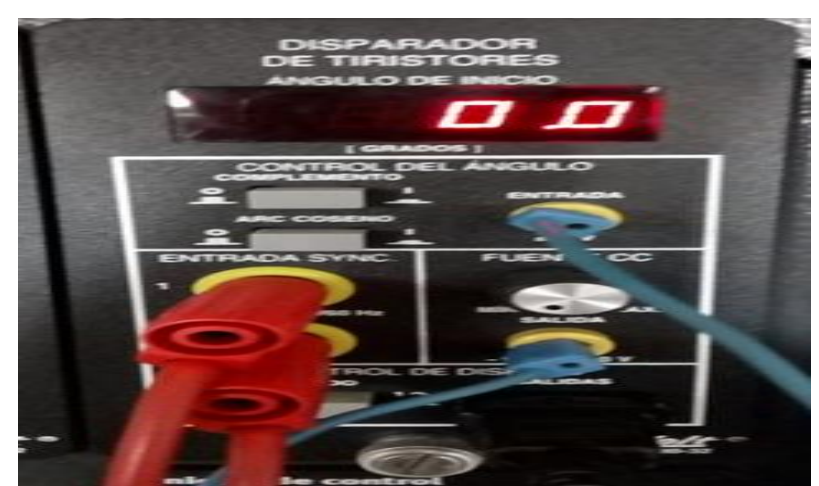

Figura 31 Angulo de disparo a $0^{\circ}$ Fuente: Elaboración Propia

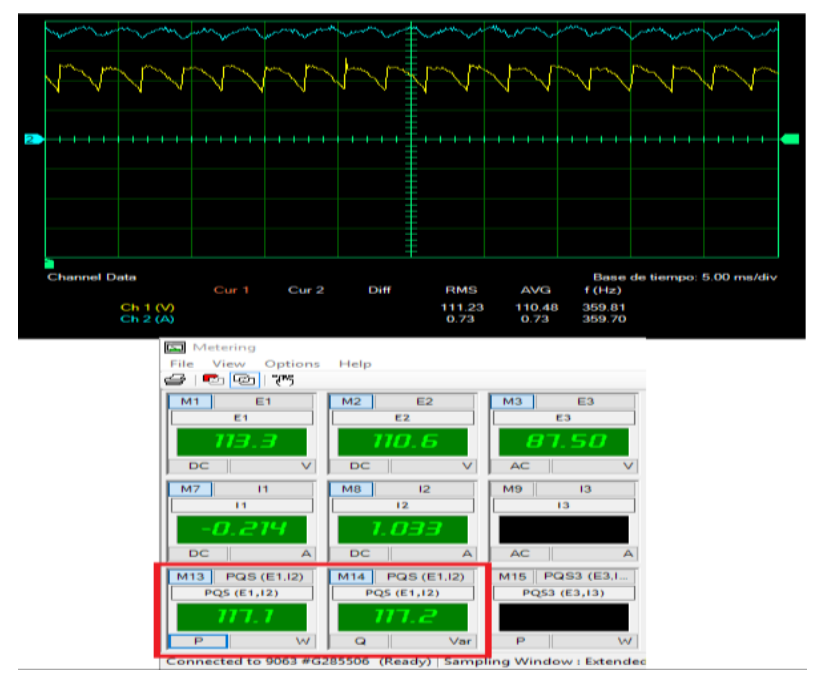

Figura 32 Gráfico y parámetros a $0^{\circ}$ Fuente: Elaboración Propia

En la figura 33 se aprecia las formas de onda de las tres fases tanto para voltaje (negro, cafe, rosa) como para corriente (azul, café, verde) cuando el ángulo de disparo está en $0^{\circ}$, en la cual se puede ver que la corriente es la más afectada por los armónicos debido a que es la que presenta mayor distorsión.

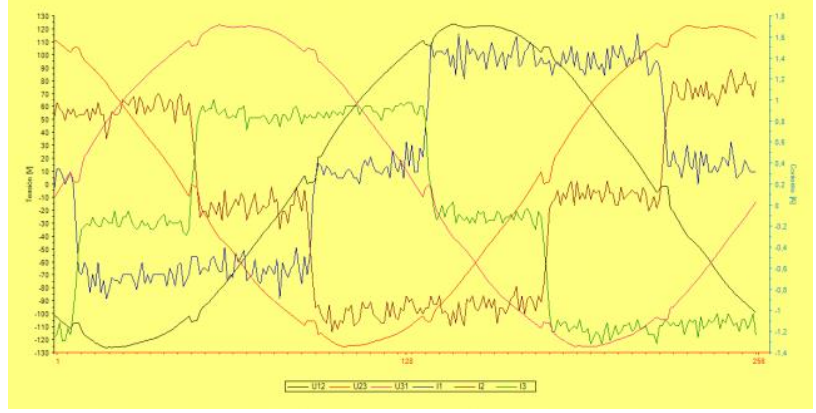

Figura 33 Formas de onda de voltaje y corriente a la salida del inversor

Fuente: Elaboración Propia

En la figura 34 se muestra el espectro de frecuencias, en el cual se puede apreciar el THD de voltaje y corriente (tonos azules), para este caso hay presencia hasta el $63^{\circ}$ armónico.

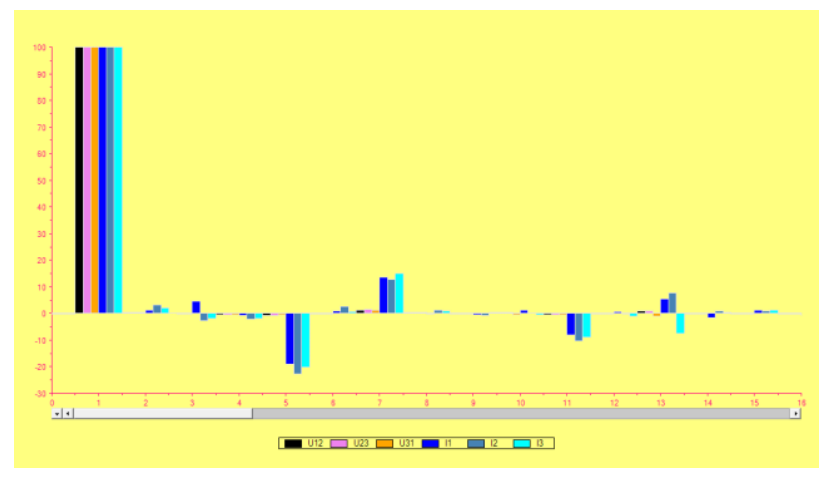

Figura 34 Espectro de frecuencias inversor trifásico Fuente: Elaboración Propia

En la figura 35 se puede observar en el multímetro el grado de THD para voltaje y corriente de cada fase que aporta el inversor.

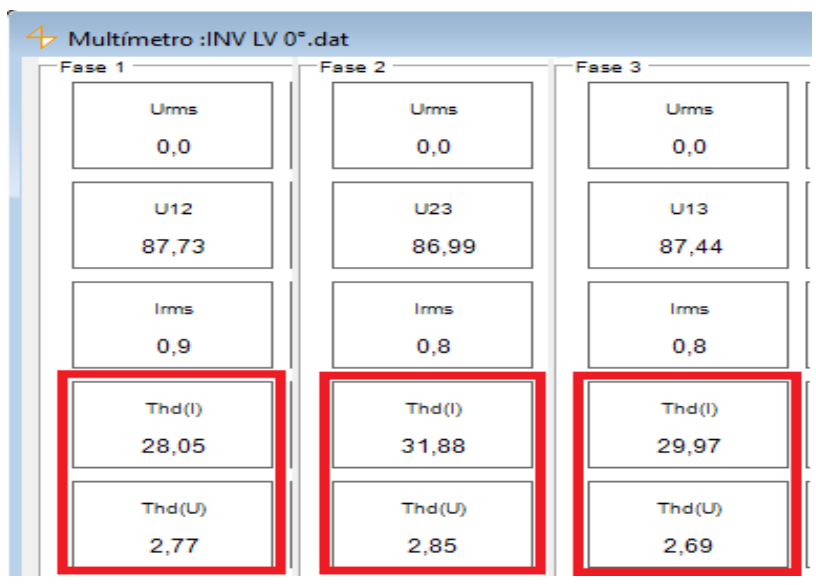

Figura 35 Valores de THD para inversor trifásico Fuente: Elaboración Propia

Se puede ver en la figura del multímetro que el THD es casi el mismo para cada fase tanto para voltaje como para corriente, siendo estos valores: 
$\begin{array}{ll}- & \text { Fase 1: } \mathrm{THD}=2.77 \% \text { y } \mathrm{THD}_{\mathrm{I}}=28.05 \% \\ - & \text { Fase 2: } \mathrm{THD}_{\mathrm{V}}=2.85 \% \text { y } \mathrm{THD}_{\mathrm{I}}=31.88 \% \\ - & \text { Fase 3: } \mathrm{THD}_{\mathrm{V}}=2.69 \% \text { y } \mathrm{THD}_{\mathrm{I}}=29.97 \%\end{array}$

\section{Micro inversor}

Este micro inversor fue configurado en el laboratorio de energías renovables, en este caso es monofásico al cual se le aplico una carga combinada la cual fue resistiva e inductiva para ver qué efectos tiene, de igual manera que en los casos anteriores, en la forma de onda de voltaje y corriente y los armónicos que aporta.

En la figura 36 se puede apreciar la forma de onda de voltaje (negro) y corriente (azul), en la cual se aprecia que la más afectada es la corriente siendo esta la que tiene mayor distorsión.

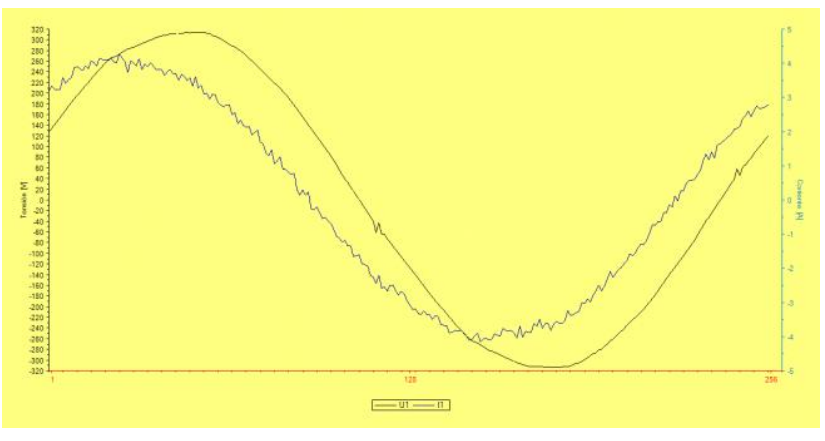

Figura 36 Formas de onda de voltaje y corriente del micro inversor

Fuente: Elaboración Propia

En la figura 37 se muestra el espectro de frecuencias en el cual se aprecia el THD en voltaje (negro) y corriente (azul), en este caso hay presencia hasta $61^{\circ}$ armónico.

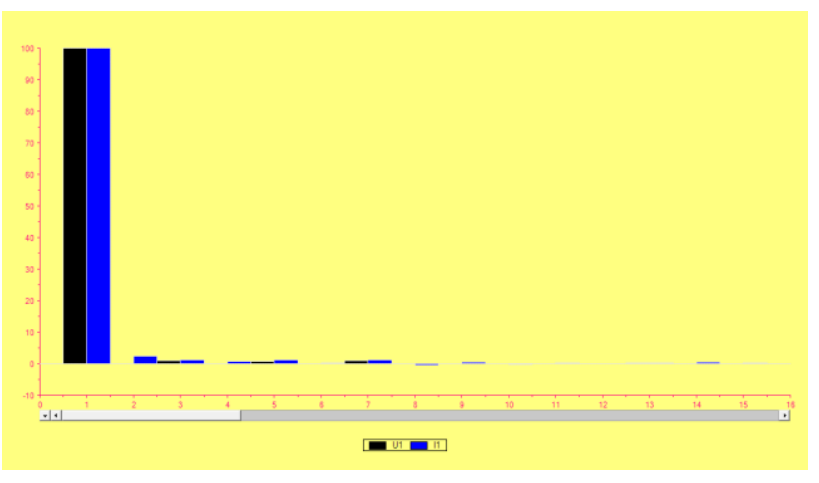

Figura 37 Espectro de frecuencias del micro inversor Fuente: Elaboración Propia

Por último, en la figura 38 se observa en el multímetro el grado de THD para voltaje y corriente, siendo para $\mathrm{THD}_{\mathrm{v}}$ un porcentaje del $1.58 \%$ y para $\mathrm{THD}_{\mathrm{i}}$ un $3.70 \%$.

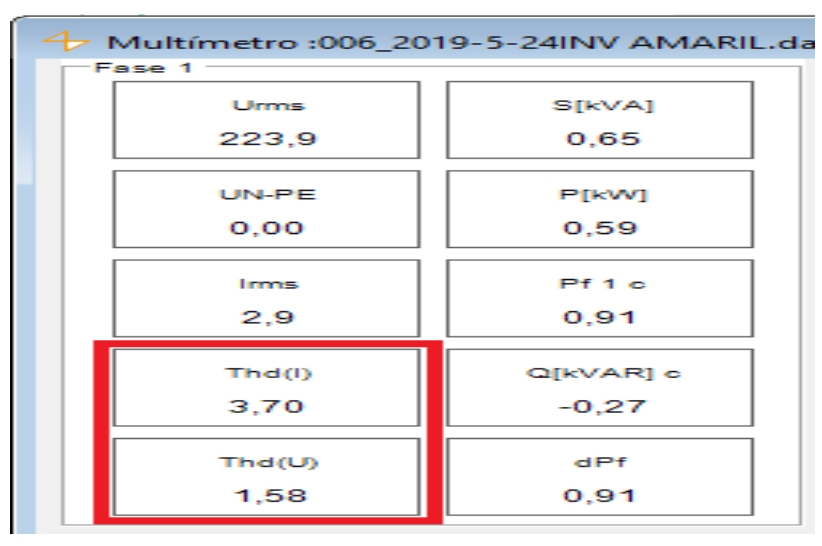

Figura 38 Valores de THD para micro inversor Fuente: Elaboración Propia

\section{Inversor KACO Blueplanet 3.0 TL1}

El cual forma parte de un SFVI a la red con capacidad de $3.6 \mathrm{KW}$ máximo, este sistema se encuentra instalado en la azotea de un edificio, figura 39.

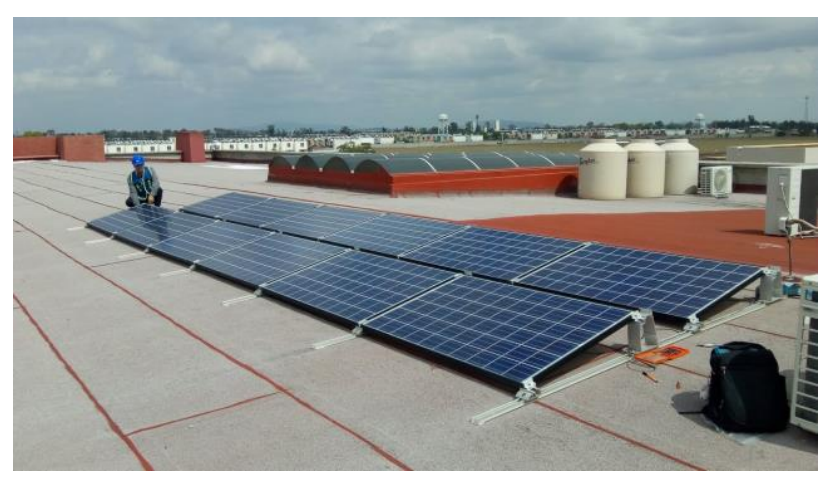

Figura 39 SFVI caso real.

Fuente: Elaboración Propia

A continuación, se muestra una tabla en la cual indica las especificaciones más importantes de dicho inversor:

\begin{tabular}{|l|l|}
\hline \multicolumn{2}{|c|}{ DC Input data } \\
\hline Max. recommended PV generator & $3600 \mathrm{~W}$ \\
\hline MPP range & $140-510 \mathrm{~V}$ \\
\hline Operating range from V to V & $125-550 \mathrm{~V}$ \\
\hline Starting voltage & $150 \mathrm{~V}$ \\
\hline Max. Input current & $2 \times 11 \mathrm{~A}$ \\
\hline Max. Short circuit current & $2 \times 13.2 \mathrm{~A}$ \\
\hline Max. Input power per tracker & $3100 \mathrm{~W}$ \\
\hline AC output data & $3000 \mathrm{VA}$ \\
\hline Rated output & $3000 \mathrm{VA}$ \\
\hline Max. Power & $230 \mathrm{~V}(1 / \mathrm{N} / \mathrm{PE})$ \\
\hline Line voltage & $50 / 60 \mathrm{~Hz}$ \\
\hline Rated frequency (range) & $13 \mathrm{~A}$ \\
\hline Rated current & $14.5 \mathrm{~A}$ \\
\hline Max. Current & $0-95 \% / 0.30$ ind. -0.30 cap. \\
\hline Cos phi & $1.42 \%$ \\
\hline THD \% & $97.2 \%$ \\
\hline General data & $3 \mathrm{~W}$ \\
\hline Max efficiency & \\
\hline Standby consumption &
\end{tabular}

Tabla 5 Especificaciones inversor KACO.

Fuente: Technical Data (KACO)

DELGADO-PONCE, Luis, VARGAS-HERNÁNDEZ, Oscar Joel, MARTÍNEZ-MAGDALENO, Arturo y MENÉNDEZ-BENAVENTE, Luis Martín. Los armónicos que aportan los sistemas fotovoltaicos interconectados a la red un problema latente. Revista de Ingeniería Eléctrica. 2019. 
El sistema eléctrico donde se conectó el inversor ya es un sistema conflictivo con alto aporte de armónicos, por lo tanto, se conectó el inversor a este sistema para ver qué tanta distorsión aporta.

\section{Pruebas a sistema eléctrico edificio}

Se analizó el sistema eléctrico del tablero F, sin SFVI conectado, para determinar niveles de armónicos. En la figura 40 se aprecian las ondas de voltaje (negro, cafe y rosa) y corriente (azul, cafe y verde) del sistema eléctrico, en el cual hay una gran aportación de armónicos, siendo las ondas de corriente las más afectadas, en el neutro (rojo) se observa que también hay una gran cantidad de armónicos.

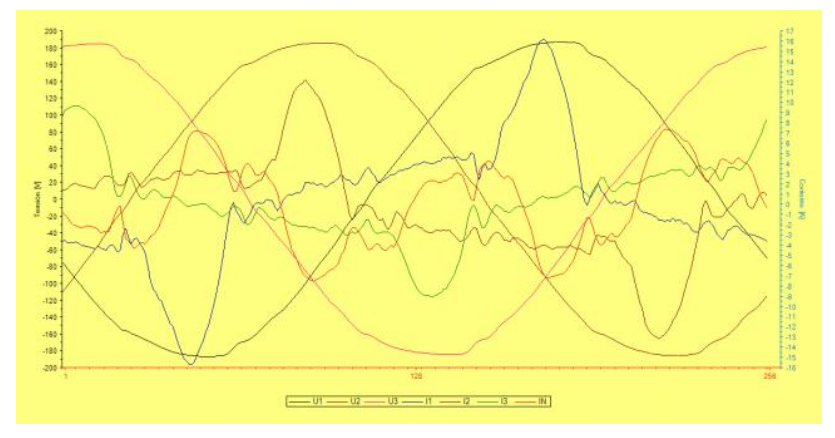

Figura 40 Formas de onda de voltaje, corriente y neutro del sistema eléctrico del edificio

Fuente: Elaboración Propia

En la figura 41 se aprecia el espectro de frecuencias en el cual se observa el nivel de THD para voltaje (negro, rosa y naranja) y corriente (tonos azules) de cada fase, además del neutro (verde) para este caso hasta el $63^{\circ}$ armónico.

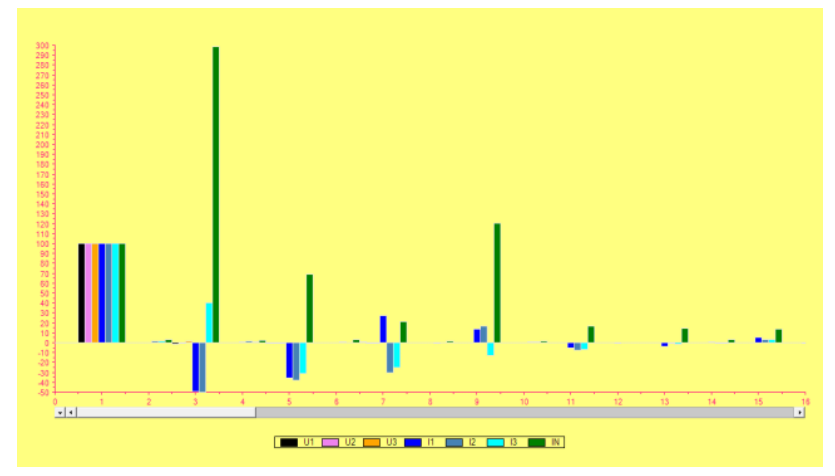

Figura 41 Espectro de frecuencias del sistema eléctrico del edificio

Fuente: Elaboración Propia

En la figura 42 se aprecia en el multímetro el grado de THD para voltaje y corriente en cada fase, siendo para cada fase los siguientes valores:
Fase 1: $\mathrm{THD}_{\mathrm{V}}=1.97 \%$ y $\mathrm{THD}_{\mathrm{I}}=68.95 \%$

Fase 2: $\mathrm{THD}_{\mathrm{V}}=1.78 \%$ y $\mathrm{THD}_{\mathrm{I}}=72.73 \%$

Fase 3: $\mathrm{THD}_{\mathrm{V}}=2.04 \%$ y $\mathrm{THD}_{\mathrm{I}}=60.53 \%$

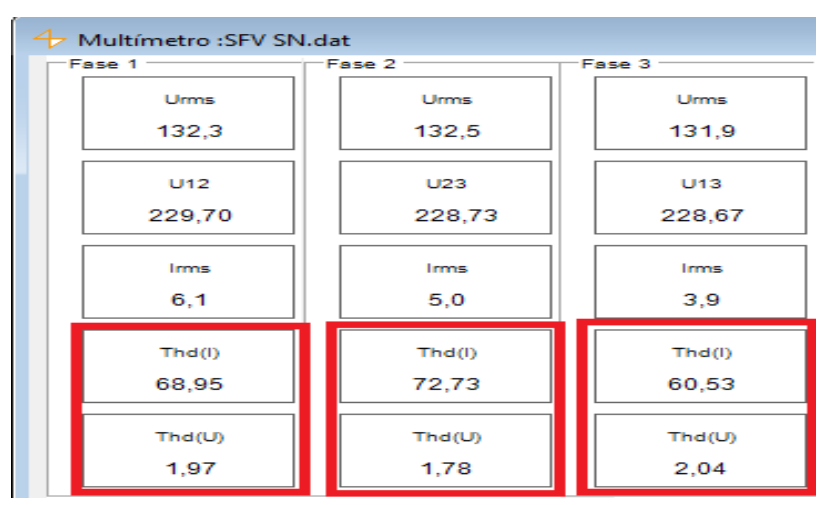

Figura 42 Valores de THD para el sistema eléctrico del edificio

Fuente: Elaboración Propia

Como el multímetro no indica el grado de THD para el neutro se tiene que obtener ese valor del indicador de THD del espectro de frecuencias, el cual indica un valor de THD de $333.66 \%$ para el neutro, en la figura 43 se muestra dicha cifra.

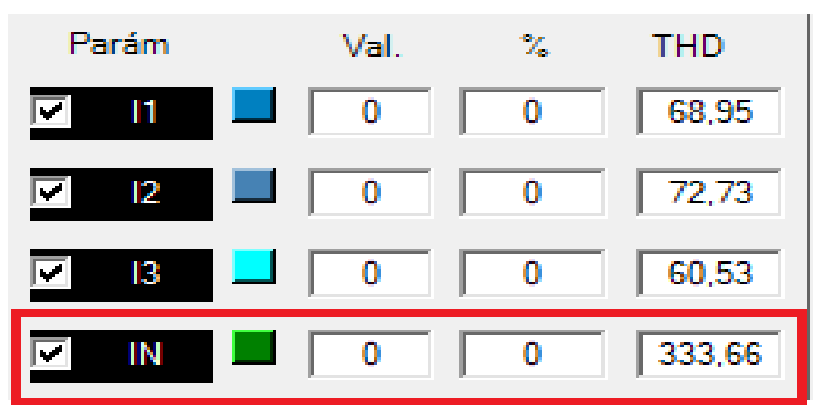

Figura 43 Valor de THD para el neutro del sistema eléctrico del edificio

Fuente: Elaboración propia

\section{Pruebas a sistema eléctrico del edificio conectando el sistema fotovoltaico}

Después de analizar el sistema eléctrico, se procedió a realizar las pruebas conectando el sistema fotovoltaico a él para determinar qué tanta distorsión aporta este.

A continuación, en la figura 44 se muestra que el inversor marcaba que el SFVI estaba generando $2.51 \mathrm{~kW}$ el día de las pruebas. 


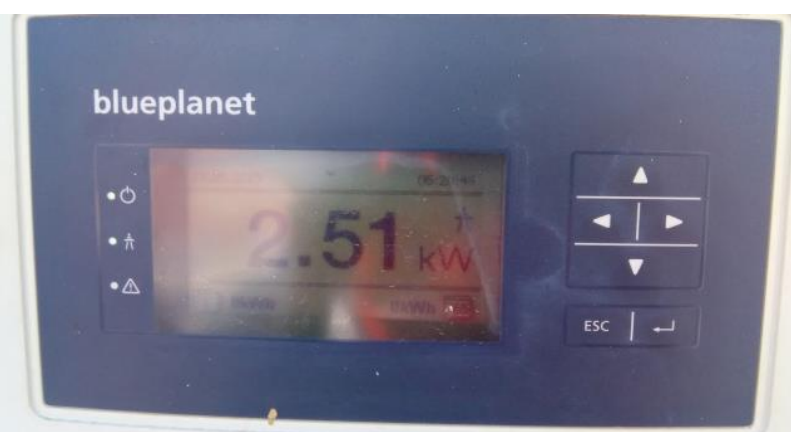

Figura 44 Nivel de kW generados por el SFVI Fuente: Elaboración Propia

En la figura 45 se puede apreciar las ondas de voltaje (negro, cafe y rosa) y corriente (azul, café y verde) del sistema eléctrico cuando ya se le ha conectado el sistema fotovoltaico y este está generando, en el cual se aprecia que hay una aportación considerable de armónicos, siendo las ondas de corriente las más afectadas, también se puede observar la onda del neutro (rojo) en el cual también hay una gran cantidad de armónicos.

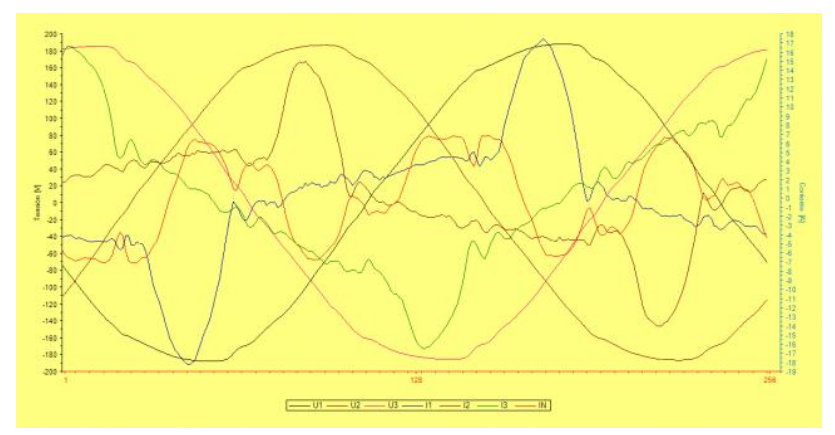

Figura 45 Formas de onda de voltaje, corriente y neutro del sistema eléctrico con el SFV conectado a él Fuente: Elaboración Propia

En la figura 46 se muestra el espectro de frecuencias en el cual se puede ver el nivel de THD para voltaje (negro, rosa y naranja) y corriente (tonos azules) de cada fase, además del neutro (verde) para este caso se tiene presencia hasta el $63^{\circ}$ armónico.

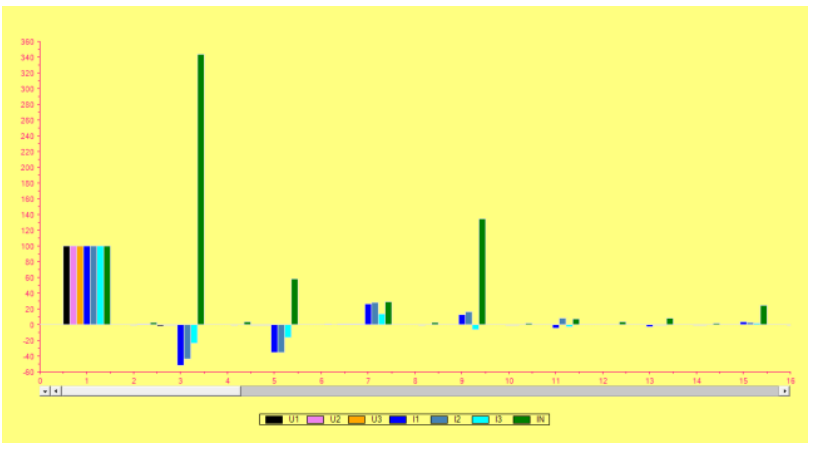

Figura 46 Espectro de frecuencias del sistema eléctrico con el SFV conectado a él

Fuente: Elaboración Propia

ISSN: 2523-2517

ECORFAN® Todos los derechos reservados
En la figura 47 se muestra en el multímetro el grado de THD para voltaje y corriente en cada fase, siendo para cada fase los siguientes valores:

$\begin{array}{ll}- & \text { Fase 1: } \mathrm{THD}_{\mathrm{V}}=2.15 \% \text { y } \mathrm{THD}_{\mathrm{I}}=70.09 \% \\ - & \text { Fase 2: } \mathrm{THD}_{\mathrm{V}}=1.73 \% \text { y } \mathrm{THD}_{\mathrm{I}}=66.15 \% \\ - & \text { Fase 3: } \mathrm{THD}_{\mathrm{V}}=2.19 \% \text { y } \mathrm{THD}_{\mathrm{I}}=33.47 \%\end{array}$

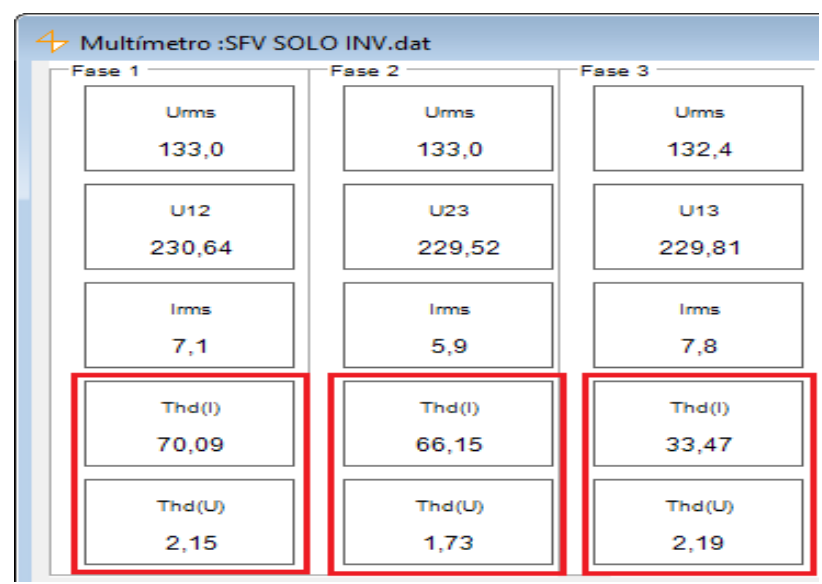

Figura 47 Valores de THD para el sistema eléctrico con el SFV conectado a él

Fuente: Elaboración Propia

El grado de THD para el neutro, marca un valor de THD de $378.11 \%$, en la figura 48 se puede apreciar dicha cifra.

\begin{tabular}{|c|c|c|c|}
\hline Parám & Val. & $\%$ & THD \\
\hline$\nabla \quad I 1$ & 0 & 0 & 70.09 \\
\hline$\square \quad 12$ & 0 & 0 & 66,15 \\
\hline$\checkmark \quad 13$ & 0 & 0 & 33,47 \\
\hline$\square \quad$ IN & 0 & 0 & 378.11 \\
\hline
\end{tabular}

Figura 48 Valor de THD para el neutro del sistema eléctrico del edificio

Fuente: Elaboración Propia

\section{Agradecimiento}

Agradecemos al Rector de la Universidad Tecnológica de Salamanca, Ing. Alfredo López Herrera, las facilidades para la elaboración del presente estudio y al CIERMMI por la invitación a participar.
DELGADO-PONCE, Luis, VARGAS-HERNÁNDEZ, Oscar Joel, MARTÍNEZ-MAGDALENO, Arturo y MENÉNDEZ-BENAVENTE, Luis Martín. Los armónicos que aportan los sistemas fotovoltaicos interconectados a la red un problema latente. Revista de Ingeniería Eléctrica. 2019. 


\section{Conclusiones}

1. Los armónicos indicados en los datos de placa de inversores no son fijos dependen de la incidencia solar, a mayor incidencia solar, mayor es la distorsión armónica.

2. La "pureza" de la forma sinodal que aportan los inversores depende de la calidad de su fabricación y diseño (generalmente precio).

3. Actualmente se usan 2 tipos de sistema de inversores, los conmutados por red y los auto conmutados.

4. Los convertidores conmutados por red son inversores que usan 6 o 12 pulsos que pueden producir armónicos enteros, este tipo de inversores no controla la potencia reactiva y consume potencia reactiva inductiva.

5. El inversor auto conmutado es modulado por ancho de pulso que usa principalmente transistores IGBT, estos inversores controlan ambas potencias activa y reactiva, por lo tanto, tienen la capacidad de proveer para la demanda en la potencia reactiva y a conmutaciones a alta frecuencia que los hacen producir altos armónicos e interarmonicos.

6. Dado que lo sistemas de generación de energía eléctrica a partir de energías limpias favorecen la generación distribuida, el aporte de armónicos es latente.

7. Solo los excedentes de potencia van a la red, por lo que para asegurar que los armónicos no se inyecten a la misma las propuestas de los SFVI no deben ser de $100 \%$ del consumo de energía eléctrica, se debe realizar el estudio para cada sistema en particular, con la premisa que todo lo generado por el SFVI sea consumido por el circuito alimentado y evitando así que no se inyecten armónicos a la red de CFE.

\section{Referencias}

Carpio, I. J., Míguez, C. J. V., \& Guirado, T. R. (2013). Alimentación de cargas críticas y calidad de la energía eléctrica. Retrieved from https://ebookcentral.proquest.com.

Cunill Solà, J. (2017). Estudio y Caracterización Armónica de las Lámparas Fluorescentes Compactas de Baja Potencia. Barcelona: Universitat Politecnica de Catelunya.

Enercen.com.mx. (2019). Sistemas Fotovoltaicos Interconectados a la Red / Enercen. (online) Available at: https://www.enercen.com.mx/sistemas-

fotovoltaicos-interconectados-a-la-red/

Gimeno, S. F. J., Seguí, C. S., \& Orts, G. S. (2011). Convertidores electrónicos: Energía solar fotovoltaica, aplicaciones y diseño. Retrieved from https://ebookcentral.proquest.com

Kaco-newenergy.com. (n.d.). KACO new energy (online) (2016). Available at: https://kaconewenergy.com. 